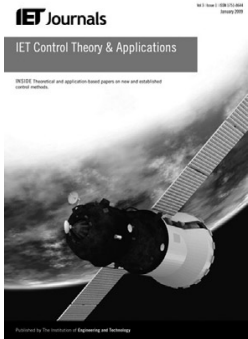

\title{
Model predictive quadrotor control: attitude, altitude and position experimental studies
}

\author{
K. Alexis ${ }^{1} \quad$ G. Nikolakopoulos ${ }^{2} \quad$ A. Tzes $^{1}$ \\ ${ }^{1}$ Department of Electrical and Computer Engineering, University of Patras, Greece \\ ${ }^{2}$ Computer Science, Electrical and Space Engineering Department, Lulea University of Technology, Sweden \\ E-mail: kostalexis@ece.upatras.gr
}

\begin{abstract}
This study addresses the control problem of an unmanned quadrotor in an indoor environment where there is lack of absolute localisation data. Based on an attached inertia measurement unit, a sonar and an optic-flow sensor, the state vector is estimated using sensor fusion algorithms. A novel switching model predictive controller is designed in order to achieve precise trajectory control, under the presence of forcible wind gusts. The quadrotor's attitude, altitude and horizontal linearised dynamics result in a set of piecewise affine models, enabling the controller to account for a larger part of the quadrotor's flight envelope while modelling the effects of atmospheric disturbances as additive-affine terms in the system. The proposed controller algorithm accounts for the state and actuation constraints of the system. The controller is implemented on a quadrotor prototype in indoor position tracking, hovering and attitude manoeuvres experiments. The experimental results indicate the overall system's efficiency in position/altitude/attitude set-point manoeuvres.
\end{abstract}

\section{Introduction}

The area of unmanned aerial vehicles (UAVs) has seen rapid growth, mainly because of the ability of UAVs to effectively carry out a wide range of applications at low costs and without putting human resources at risk. Nowadays, UAVs are being used in several types of missions including search and rescue missions [1], wild fire surveillance [2], monitoring over nuclear reactors [3], power plants inspection [4], agricultural services [5], mapping and photographing [5], marine operations [6], battle damage assessment [7], border interdiction prevention [8] and law enforcement [9].

The aforementioned extended set of possible applications imposes new demands in the areas of control and navigation in order to design unmanned systems capable of operating in harsh environments and coping with complex missions. Both in manned and unmanned aviation, helicopters and general rotorcrafts have been proven one of the best solutions because of some important capabilities, including vertical take-off and landing and aggressive manoeuvrability. However, rotorcraft UAVs pose significant scientific and engineering problems that must be addressed in order to be able to fly autonomously and efficiently. These machines are characterised by aggressive dynamics because of their low inertia moments and are subject to complex aerodynamic effects that affect their flight. This sets very strict requirements in terms of state estimation and controller implementation at high update rates. However, low-cost onboard sensory systems are noisy and present drifting characteristics, and thus the control problem becomes more complex. Additionally, there are hard constraints in the actuation/propulsion systems that further complicate the control design problem.

Consequently, the problem of electro-mechanical design and autonomous control of these systems is challenging. This problem becomes even more demanding if the perturbation effects of atmospheric disturbances are taken into account in order to develop systems able to navigate in actual mission environments. Thus, novel control laws should: (a) take into account the constraints of the system, and (b) produce efficient control actions.

In the area of unmanned quadrotors, the problem of control design has primarily focused in the following areas: (a) proportional-integral-differential (PID) controllers, PID controllers augmented with angular acceleration feedback and linear quadratic (LQ)-regulators [10-12], (b) nonlinear control methods including sliding mode controllers [13], backstepping control approaches [14-16] and integral predictive-nonlinear $H_{\infty}$ control [17], (c) dynamic inversionbased techniques [18], (d) constrained finite time optimal control schemes $[19,20]$ and (e) model predictive attitude control [21]. In addition, in most of the existing literature of rotorcrafts, research efforts on the effects of the environmental disturbances, such as in [22, 23], have focused primarily in simulations and not in experimental studies.

The main contribution of this paper is the introduction and experimental verification of a new trajectory control methodology for a quadrotor. The proposed novel control strategy is based on a piecewise affine (PWA) dynamic modelling approach, and on a switching model predictive control (SMPC) [24-26] design scheme. More specifically, the contributions of this paper include: (a) the PWA 
modelling of the attitude and translational dynamics of the quadrotor that enable the development of switching control actions for a larger part of the helicopter's flight envelope, (b) the modelling of the effects induced by wind gusts as affine output disturbances, (c) the development of an SMPC that accounts for the state and actuation constraints of the quadrotor, and (d) the application of the proposed control scheme in an indoor environment, where there is lack of absolute localisation data (i.e. GPS, positioning from external cameras etc.) and the quadrotor's translational and rotational motion vectors are estimated by using sensor fusion algorithms on data sets obtained from an inertia measurement unit (IMU), an altitude sonar and an optic-flow sensor.

The efficiency of the overall proposed scheme, is experimentally being evaluated in multiple flight test cases, including: (a) position hold, (b) trajectory tracking, (c) hovering and (d) aggressive attitude regulation manoeuvering. The experiments were performed using a new experimental prototype of an unmanned quadrotor (UPATcopter), illustrated in Fig. 1. Special attention has been given to the design and development of this prototype, in order to design a UAV, capable of utilising computationally intensive control laws, utilising a wide set of sensors, communicating through wireless networks and ensuring easy upgradeability.

This paper is an extension and a significant breakthrough of the ideas presented in [21] where the attitude problem was addressed using a preliminary version of the SMPCapproach and verified on a completely different experimental set-up. The main contributions of this paper include: (a) the design of an SMPC-scheme for both the translational and attitude dynamics of the quadrotor is based on a PWA modelling of the six-degrees of freedom (6-DOF) dynamics that takes into account a significant subset of the couplings that rule the system's behaviour, (b) it is the first time that an MPC approach is being designed and experimental verified for the complete trajectory and attitude control of a quadrotor, (c) the coupled tuning of the attitude and translational controllers since the overall control problem is naturally coupled both in the dynamics and the aerodynamics sense, (d) the design and implementation of a totally different experimental set-up including complete in-house design of a new quadrotor prototype with highlevel onboard state estimation capabilities, computational power, modular communication connectivity and actuation efficiency, (e) the implementation of all control and estimation algorithms onboard in high update rates as opposed to the off-board low-rate implementation of a

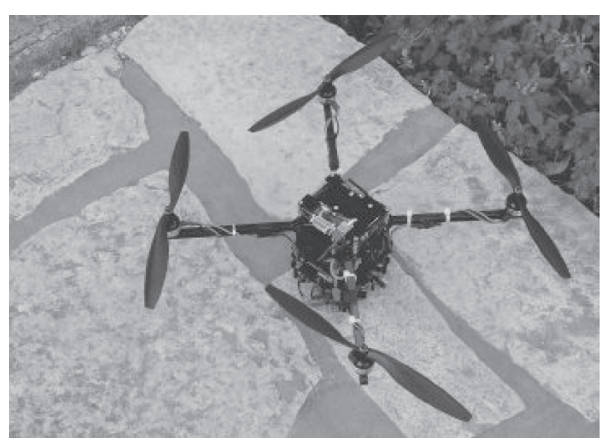

Fig. 1 UPATcopter: university of Patras' experimental quadrotor prototype much lower complexity scheme in [21], and (f) a new modelling approach even for the attitude dynamics that uses integral state augmentation for the attitude PWA affine systems and PWA error dynamics for the translational dynamics. The rest of this paper is structured as follows. In Section 2, the modelling approach for the attitude, altitude and horizontal $x-y$ motion dynamics of the quadrotor is presented, followed by the mathematical formulation of the physical, mechanical, state and input constraints and the effects of wind disturbances. In Section 3, the data fusion concept for using the data from the integrated sensor system is presented. In Section 4, the design and the development of the SMPC scheme is analysed for the quadrotor's 6-DOF set-point control problem. Experimental results are presented in order to highlight the overall efficiency of the proposed controller in Section 5. Finally, in Section 6 the conclusions are drawn.

\section{Quadrotor dynamics}

The quadrotor's motion is governed by the lift forces, produced by the rotating propeller blades, whereas the translational and rotational motions are achieved by means of difference in the counter rotating blades. Specifically, the forward motion is achieved by the difference in the lift force produced from the front and the rear rotors' velocity, the sidewards-motion by the difference in the lift force from the two lateral rotors, whereas the yaw motion is produced by the difference in the counter-torque between the two pairs of rotors front-right and back-left. Finally, motion at the perpendicular axis is produced by the total rotor thrust.

The model of the quadrotor utilised in this paper assumes that the structure is rigid and symmetrical, the centre of gravity and the body-fixed frame (BFF) origin coincide, the propellers are rigid and the thrust and drag forces are proportional to the square of propeller's speed. The BFF $\mathbf{B}=\left[\begin{array}{lll}B_{1}, & B_{2}, & B_{3}\end{array}\right]^{\mathrm{T}}$ and the earth-fixed frame (EFF) $\mathbf{E}=$ $\left[E_{x}, E_{y}, E_{z}\right]^{\mathrm{T}}$ are presented in Fig. 2.

Special attention should be paid in the difference between the body rates measured $p, q, r$ in BFF and the Tait-Bryan angle rates expressed in EFF. The transformation matrix from $\left[\begin{array}{lll}\dot{\phi} & \dot{\theta} & \dot{\psi}\end{array}\right]^{\mathrm{T}}$ to $\left[\begin{array}{lll}p & q & r\end{array}\right]^{\mathrm{T}}$ is given by

$$
\left[\begin{array}{l}
p \\
q \\
r
\end{array}\right]=\left[\begin{array}{ccc}
1 & 0 & -\sin \theta \\
0 & \cos \phi & \sin \phi \cos \theta \\
0 & -\sin \phi & \cos \phi \cos \theta
\end{array}\right]\left[\begin{array}{c}
\dot{\phi} \\
\dot{\theta} \\
\dot{\psi}
\end{array}\right]
$$

Moreover, the rotation of the quadrotor's body must also be compensated during position control. The compensation is

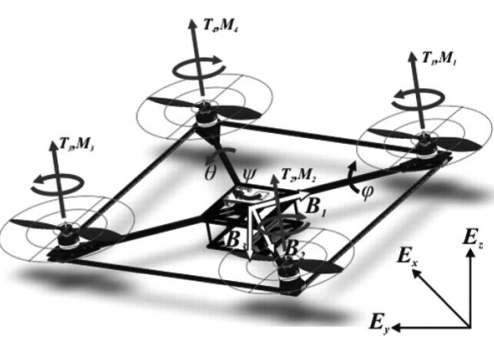

Fig.2 Quadrotor helicopter frame system configuration 
achieved using the transpose of the rotation matrix

$$
\begin{aligned}
R(\phi, \theta, \psi) & =R(x, \phi) R(y, \theta) R(z, \psi) \\
R(x, \phi) & =\left[\begin{array}{ccc}
1 & 0 & 0 \\
0 & \cos \phi & -\sin \theta \\
0 & \sin \phi & \cos \phi
\end{array}\right], \\
R(y, \theta) & =\left[\begin{array}{ccc}
\cos \theta & -\sin \psi & 0 \\
\sin \psi & \cos \psi & 0 \\
0 & 0 & 1
\end{array}\right], \\
R(z, \psi) & =\left[\begin{array}{ccc}
\cos \psi & -\sin \psi & 0 \\
\sin \psi & \cos \psi & 0 \\
0 & 0 & 1
\end{array}\right]
\end{aligned}
$$

The main aerodynamic forces and moments acting on the quadrotor, during a hovering flight segment, correspond to the thrust $(\mathbf{T})$, the hub forces $(\mathbf{H})$ and the drag moment (Q) because of vertical, horizontal and aerodynamic forces, respectively, followed by the rolling moment $(\mathbf{R})$ related to the integration, over the entire rotor, of the lift of each section, acting at a given radius. An extended formulation of these forces and moments can be found in [14, 27]. The nonlinear dynamics is described by the following equation [21]

$\dot{\mathbf{X}}=\left[\begin{array}{c}\dot{\phi} \\ \ddot{\phi} \\ \dot{\theta} \\ \ddot{\theta} \\ \dot{\psi} \\ \ddot{\psi} \\ \hline \dot{z} \\ \ddot{z} \\ \dot{x} \\ \ddot{x} \\ \dot{y} \\ \ddot{y}\end{array}\right]=\left[\begin{array}{c}\dot{\phi} \\ \dot{\theta} \dot{\psi} \frac{I_{y y}-I_{z z}}{I_{x x}}+\dot{\theta} \frac{J_{\mathrm{r}}}{I_{x x}} \Omega_{\mathrm{r}}+\frac{l_{\mathrm{a}}}{I_{x x}} U_{2} \\ \dot{\phi} \dot{\psi} \frac{I_{z z}-I_{x x}}{I_{y y}}-\dot{\phi} \frac{J_{\mathrm{r}}}{I_{y y}} \Omega_{\mathrm{r}}+\frac{l_{\mathrm{a}}}{I_{y y}} U_{3} \\ \dot{\psi} \\ \dot{\theta} \dot{\phi} \frac{I_{x x}-I_{y y}}{I_{z z}}+\frac{1}{I_{z z}} U_{4} \\ \dot{z} \\ g-(\cos \phi \cos \theta) U_{1} / m_{\mathrm{s}} \\ \dot{x} \\ u_{x} U_{1} / m_{\mathrm{s}} \\ \dot{y} \\ u_{y} U_{1} / m_{\mathrm{s}}\end{array}\right]+\left[\begin{array}{c}0 \\ \tilde{W}_{1} \\ 0 \\ \tilde{W}_{2} \\ 0 \\ \tilde{W}_{3} \\ 0 \\ \tilde{W}_{4} \\ 0 \\ \tilde{W}_{5} \\ 0 \\ \tilde{W}_{6}\end{array}\right]$

$$
\begin{gathered}
\mathbf{U}=\left[\begin{array}{l}
U_{1} \\
U_{2} \\
U_{3} \\
U_{4} \\
\Omega_{\mathrm{r}}
\end{array}\right]=\left[\begin{array}{c}
b\left(\Omega_{1}^{2}+\Omega_{2}^{2}+\Omega_{3}^{2}+\Omega_{4}^{2}\right) \\
b\left(-\Omega_{2}^{2}+\Omega_{4}^{2}\right) \\
b\left(\Omega_{1}^{2}-\Omega_{3}^{2}\right) \\
d\left(-\Omega_{1}^{2}+\Omega_{2}^{2}-\Omega_{3}^{2}+\Omega_{4}^{2}\right) \\
-\Omega_{1}+\Omega_{2}-\Omega_{3}+\Omega_{4}
\end{array}\right] \\
{\left[\begin{array}{l}
u_{x} \\
u_{y}
\end{array}\right]=\left[\begin{array}{l}
\cos \phi \sin \theta \cos \psi+\sin \phi \sin \psi \\
\cos \phi \sin \theta \sin \psi-\sin \phi \cos \psi
\end{array}\right]}
\end{gathered}
$$

Table 1 Quadrotor model parameters

\begin{tabular}{ll}
\hline$I_{x x}\left(I_{y y}\right)\left[I_{z z}\right]$ & moment of inertia of the quadrotor about the \\
$I_{\mathrm{a}}$ & $E_{x}\left(E_{y}\right)\left[E_{z}\right]$ axis \\
$b, d$ & quadrotor's arm length \\
$J_{\mathrm{r}}$ & thrust, drag coefficients \\
& moment of inertia of the rotor about its axis of \\
& rotation \\
\hline
\end{tabular}

where $\mathbf{U}$ is the input vector consisting of $U_{1}$ (total thrust), and $U_{2}, U_{3}, U_{4}$ which are related to the rotations of the quadrotor, and $\Omega_{\mathrm{r}}$ representing the overall residual propeller angular speed, while $\Omega_{1}, \ldots, \Omega_{4}$ correspond to the propellers' angular speeds, $\mathbf{X}$ is the state vector that consists of: (a) the translational components $\xi=[x, y, z]^{\mathrm{T}}$ and their derivatives, and (b) the rotational components $\dot{\eta}=[\dot{\phi}, \dot{\theta}, \dot{\psi}]^{\mathrm{T}}$ and their derivatives, $m_{\mathrm{s}}$ is the total mass of the quadrotor, $g=9.81 \mathrm{~m} / \mathrm{s}^{2}$ is the gravitational acceleration. The effects of the external disturbances, are accounted by the additive disturbance vector $\tilde{\mathbf{W}}$. The rest of the parameters in (3) and (4) are listed in Table 1.

Under the assumption of small velocities [12, 27] the attitude dynamics in (3) are decoupled from the translational dynamics.

\subsection{Attitude dynamics}

In order to derive the PWA representation of the quadrotor's linearised attitude dynamics, small attitude perturbations $\delta_{\lambda}$, with $\lambda \in \mathbb{Z}^{+}$, around the operating points $\left[0, \dot{\phi}^{\circ, \lambda}, 0, \dot{\theta}^{\circ, \lambda}, 0, \dot{\psi}^{\circ, \lambda}\right]^{\mathrm{T}}$ are assumed.

In order to account for set-point control purposes, the attitude state vector is augmented with the integrals of the roll, pitch and yaw angles. The resulting PWA-linearised dynamics is an extension of the state space matrices presented in [21]

$$
\begin{aligned}
& \dot{\mathbf{x}}_{\eta}=\mathbf{A}_{\eta}^{\lambda} \mathbf{x}_{\eta}+\mathbf{B}_{\eta}^{\lambda} \mathbf{u}_{\eta}+\tilde{\mathbf{W}}_{\eta} \\
& \mathbf{x}_{\eta}=\left[\phi, \delta \dot{\phi}^{\lambda}, \int \phi \mathrm{d} t, \theta, \delta \dot{\theta}^{\lambda}, \int \theta \mathrm{d} t, \psi, \delta \dot{\psi}^{\lambda}, \int \psi \mathrm{d} t\right]^{\mathrm{T}} \\
& \mathbf{u}_{\eta}=\left[\delta U_{1}, \delta U_{2}, \delta U_{3}, \delta U_{4}, \delta \Omega_{\mathrm{r}}\right]^{\mathrm{T}} \\
& \tilde{\mathbf{W}}_{\eta}=\left[0, \delta W_{1}, 0,0, \delta W_{2}, 0,0, \delta W_{3}, 0\right]^{\mathrm{T}}
\end{aligned}
$$

(see (7))

$$
\mathbf{A}_{\eta}^{\lambda}=\left[\begin{array}{ccccccccc}
0 & 1 & 0 & 0 & 0 & 0 & 0 & 0 & 0 \\
0 & 0 & 0 & 0 & \frac{I_{y y}-I_{z z}}{I_{x x}} \dot{\psi}^{\circ, \lambda} & 0 & 0 & \frac{I_{y y}-I_{z z}}{I_{x x}} \dot{\theta}^{\circ, \lambda} & 0 \\
1 & 0 & 0 & 0 & 0 & 0 & 0 & 0 & 0 \\
0 & 0 & 0 & 0 & 1 & 0 & 0 & 0 & 0 \\
0 & \frac{I_{z z}-I_{x x}}{I_{y y}} \dot{\psi}^{\circ, \lambda} & 0 & 0 & 0 & 0 & 0 & \frac{I_{y y}-I_{x x}}{I_{y y}} \dot{\phi}^{\circ, \lambda} & 0 \\
0 & 0 & 0 & 1 & 0 & 0 & 0 & 0 & 0 \\
0 & 0 & 0 & 0 & 0 & 0 & 0 & 1 & 0 \\
0 & \frac{I_{x x}-I_{y y}}{I_{z z}} \dot{\theta}^{\circ, \lambda} & 0 & 0 & \frac{I_{x x}-I_{y y}}{I_{z z}} \dot{\phi}^{\circ, \lambda} & 0 & 0 & 0 & 0 \\
0 & 0 & 0 & 0 & 0 & 0 & 1 & 0 & 0
\end{array}\right]
$$




$$
\mathbf{B}_{\eta}^{\lambda}=\left[\begin{array}{ccccc}
0 & 0 & 0 & 0 & 0 \\
0 & \frac{l_{\mathrm{a}}}{I_{x x}} & 0 & 0 & \frac{J_{\mathrm{r}}}{I_{x x}} \dot{\theta}^{\circ, \lambda} \\
0 & 0 & 0 & 0 & 0 \\
0 & 0 & 0 & 0 & 0 \\
0 & 0 & \frac{l_{\mathrm{a}}}{I_{y y}} & 0 & \frac{J_{\mathrm{r}}}{I_{y y}} \dot{\theta}^{\circ, \lambda} \\
0 & 0 & 0 & 0 & 0 \\
0 & 0 & 0 & 0 & 0 \\
0 & 0 & 0 & \frac{1}{I_{z z}} & 0 \\
0 & 0 & 0 & 0 & 0
\end{array}\right]
$$

cascade control approaches have been proposed by other authors as in [15].

\section{Optic-flow and IMU/sonar data fusion}

Assuming a sampling period $T_{\mathrm{s}}^{\eta}$, (6) can be discretised resulting in order to compute the SMPC scheme

$$
\mathbf{x}_{\eta}(k+1)=\overline{\mathbf{A}}_{\eta}^{\lambda} \mathbf{x}_{\eta}(k)+\overline{\mathbf{B}}_{\eta}^{\lambda} \mathbf{u}_{\eta}(k)+\tilde{\mathbf{w}}_{\eta}
$$

where $k$ corresponds to the sample index.

\subsection{Translational dynamics}

Let $\mathbf{x}_{E_{z}}=\left[\tilde{z}(t), \dot{\tilde{z}}(t), \int \tilde{z}(t) \mathrm{d} t\right]^{\mathrm{T}}, \tilde{z} \triangleq z-z^{r}$ be the altitude error dynamics vector, with respect to the $z^{r}$ reference altitude. The discretised (with a sampling period $T_{\mathrm{s}}^{t} \neq T_{\mathrm{s}}^{\eta}$ ) altitude error dynamics is [17]

$$
\begin{aligned}
\mathbf{x}_{E_{z}}(k+1)= & \overline{\mathbf{A}}_{E_{z}} \mathbf{x}_{E_{z}}(k)+\overline{\mathbf{B}}_{E_{z}}^{\mathbf{v}} u_{E_{z}}(k)+\tilde{\mathbf{w}}_{E_{z}} \\
= & {\left[\begin{array}{ccc}
1 & T_{\mathrm{s}}^{t} & 0 \\
0 & 1 & 0 \\
T_{\mathrm{s}}^{t} & 0 & 1
\end{array}\right] \mathbf{x}_{E_{z}}(k)+\left[\begin{array}{c}
0 \\
\frac{T_{\mathrm{s}}^{t}}{m_{\mathrm{s}}} \cos \theta^{\circ, v} \cos \phi^{\circ, v} \\
0
\end{array}\right.} \\
& \times\left[\delta U_{1}\right]+\tilde{\mathbf{w}}_{E_{z}}
\end{aligned}
$$

where the nominal operating points $\theta^{\circ, v}$ and $\phi^{\circ, v}$ affect only the $\overline{\mathbf{B}}_{E_{z}}^{\mathbf{v}}$ term. Overall, the error altitude dynamics is cast as a switching PWA system with $v$ acting as the switching index.

Let $\tilde{x} \triangleq x-x^{r}$ and $\tilde{y} \triangleq y-y^{r}$ and $\mathbf{x}_{E_{x} E_{y}}=[\tilde{x}(t), \dot{\tilde{x}}(t)$, $\left.\int \tilde{x}(t) \mathrm{d} t, \tilde{y}, \dot{\tilde{y}}, \int \tilde{y}\right]^{\mathrm{T}}$. The discrete representation for the $E_{x}-$ $E_{y}$ quadrotor's horizontal integral error dynamics, assuming the same sampling period $T_{\mathrm{s}}^{t}$ is [17]

$$
\begin{aligned}
\mathbf{x}_{E_{x} E_{y}}(k+1)= & \overline{\mathbf{A}}_{E_{x} E_{y}} \mathbf{x}_{E_{x} E_{y}}(k)+\overline{\mathbf{B}}_{E_{x} E_{y}}^{p} u_{E_{x} E_{y}}(k)+\tilde{\mathbf{w}}_{E_{x} E_{y}} \\
= & {\left[\begin{array}{c|c}
\overline{\mathbf{A}}_{E_{z}} & \mathbf{0}_{3 \times 3} \\
\hline \mathbf{0}_{3 \times 3} & \overline{\mathbf{A}}_{E_{z}}
\end{array}\right] \mathbf{x}_{E_{x} E_{y}}(k) } \\
& +\left[\begin{array}{cc}
0 & 0 \\
\frac{T_{\mathrm{s}}^{t} U_{1}^{\circ, p}(k)}{m_{\mathrm{s}}} & 0 \\
0 & 0 \\
0 & 0 \\
0 & \frac{T_{\mathrm{s}}^{t} U_{1}^{\circ, p}(k)}{m_{\mathrm{s}}} \\
0 & 0
\end{array}\right]\left[\begin{array}{c}
u_{x} \\
u_{y}
\end{array}\right]+\tilde{\mathbf{w}}_{E_{x} E_{y}}
\end{aligned}
$$

Similar to the altitude subsystem, the term $\overline{\mathbf{B}}_{E_{x} E_{y}}^{p}$ in (12) is switching, with respect to the total thrust $U_{1}^{\circ, p}$. Considering multiple nominal $U_{1}^{\circ, p}$ operation points, (12) can be cast as a PWA system, with $p \in \mathbb{Z}^{+}$, the switching rule. Similar
Complete indoor state estimation has been implemented by employing data fusion from multiple sensor systems. A commercial IMU (Xsens Mti-G [28]) is utilised for implementing a sophisticated variant of the extended Kalman filter (EKF) algorithm. The IMU provides accurate estimations of $[\phi, \dot{\phi}, \theta, \dot{\theta}, \psi, \dot{\psi}]^{\mathrm{T}}$ and calibrated translational acceleration measurements expressed in the EFF. Through data fusion of these data with the a-posteriori measurements of the sonar using a two-state EKF, the precise estimation of $[z, \dot{z}]^{\mathrm{T}}$ expressed in EFF can be achieved.

The estimation problem of horizontal translation is challenging and typically is solved using stationary fixed cameras that observe the rotorcraft's motion and provide absolute $[x, y]$ measurements, or onboard measurements of the position changes. The latter option was selected within the framework of this work. In order to provide $[\delta x, \delta y]$ measurements of the horizontal motion deviation, an optic-flow device for the flying quadrotor was employed. The developed optic-flow system is based on the lowcost Tam2 $16 \times 16$ vision chip [29]. The Tam series of vision chips are low-resolution image sensors performing low-level analogue processing using VLSI circuitry. The pixels have a logarithmic response to light intensity and use a basic four-transistor readout, enabling operation over a large range of intensity values. The Tam 2 vision chip has a $84 \mu \mathrm{m}$ fixed pitch, and a $1.34 \mathrm{~mm} \times 1.34 \mathrm{~mm}$ focal plane size, and an adapted $75^{\circ}$ field of view lens. The voltage drop across the transistors of the vision chip will be a logarithmic function of the current flowing through them, and thus a logarithmic function of the light intensity. A large range of light intensities may thus be compressed within a manageable voltage swing, thus providing the capability to effectively operate indoors despite reduced lighting conditions.

Optic-flow data are computed based on the aforementioned Tam2 vision chip and the image interpolation algorithm (I2A) [30]. In I2A, the parameters of global motion in a given region of the image can be estimated by a singlestage, non-iterative process. Specifically, the position of a newly acquired image is interpolated in relation to a set of older reference images. The I2A estimates the global motion of a whole image region covering a wider field of view, thus displaying no dependency on image contrast, nor on spatial frequency, as long as some image gradient is present somewhere in the considered image region.

The I2A algorithm is implemented in both $E_{x}, E_{y}$-axis. Let $I(n)$ denote the grey level of the $n$th pixel in one row of the pixel array of the vision chip. The algorithm computes the amplitude of the translation $s^{d}$ between a reference image region $I(n, t)$ captured at time $t$, called reference image, and a subsequent image $I(n, t+\Delta t)$ captured after a small period of time $\Delta t$. It is assumed that, for small displacements of the image, $I(n, t+\Delta t)$ can be approximated by $\hat{I}(n+$ $\Delta t)$, which is a weighted linear combination of the reference image and of two shifted versions $I(n \pm q, t)$ of that same image

$$
\hat{I}(n, t+\Delta t)=I(n, t)+s^{d} \frac{I(n-q, t)-I(n+q, t)}{2 q}
$$




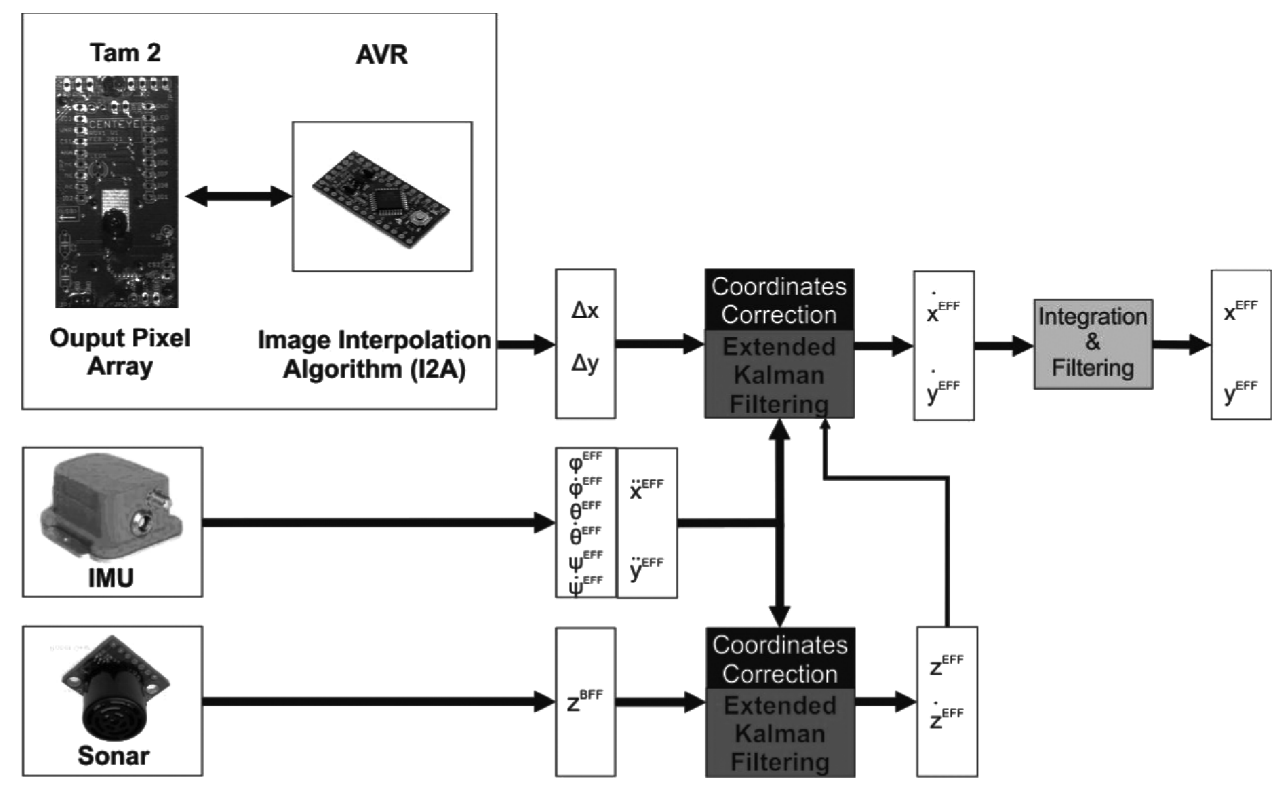

Fig. 3 Integrated indoor sensor system for quadrotor state estimation

where $q$ is a small shift in pixels. The image displacement $s^{d}$ is computed as the quantity that minimises the mean square error (MSE) $E^{\text {mse }}$ between the estimated image and the new image [31]

$$
s^{d}=2 q \frac{\sum_{n}[I(n, t+\Delta t)-I(n, t)][I(n-q, t)-I(n+q, t)]}{\sum_{n}[I(n-q, t)-I(n+q, t)]^{2}}
$$

In the developed optic-flow system, this process is applied both row- and column-wise, thus providing twodimensional (2D) optic-flow motion measurements. The measured displacements at $E_{x}, E_{y}$-axis are denoted as $\delta x^{m}, \delta y^{m}$, respectively.

Although, the optic-flow sensor will measure deviations in the $E_{x}, E_{y}$ axes, these measurements must be corrected in order to compensate for false position deviation measurements because of rolling and pitching of the quadrotor. The corrected $\delta x^{\prime}, \delta y^{\prime}$ measurements from $\delta x^{m}, \delta y^{m}$ which also account for the yawing of the vehicle can be computed as

$$
\begin{aligned}
\delta x & =\delta x^{m}+\dot{\phi} T_{\mathrm{s}}^{t} \frac{N_{\text {pixels }} c}{a_{\mathrm{f}}} \\
\delta y & =\delta y^{m}-\dot{\theta} T_{\mathrm{s}}^{t} \frac{N_{\text {pixels }} c}{a_{\mathrm{f}}} \\
\delta x^{\prime} & =\cos \psi \cdot \delta x+\sin \psi \cdot \delta y \\
\delta y^{\prime} & =\cos \psi \cdot \delta y+\sin \psi \cdot \delta x
\end{aligned}
$$

where $N_{\text {pixels }}$ is the number of pixels of the optic-flow sensor, $a_{\mathrm{f}}=75^{\circ}$ the field of view of the used lens and $c$ an arbitrary constant. It should be noted that despite the fact that Tam2 vision chip provided a $16 \times 16$ pixel array only the subset of $12 \times 12$ pixels were used as input in the I2A algorithm $\left(N_{\text {pixels }}=12\right)$.

Once the corrected deviations $\delta x, \delta y$ have been computed, a couple of two-state EKFs that make use of the aforementioned position variation measurements and accelerometers' data that are provided by the IMU are being used in order to accurately estimate the quadrotor's linear velocity. Let $v_{x}=\delta_{x}^{\prime} / T_{\mathrm{s}}^{t}, v_{y}=\delta_{y}^{\prime} / T_{\mathrm{s}}^{t}$ be the absolute velocity measurements, and $v \rightarrow(x, y)$. Also formulate the vectors $p^{x}=\left[\begin{array}{ll}v_{x} & \dot{v}_{x}\end{array}\right]^{\mathrm{T}}, p^{y}=t\left[\begin{array}{ll}v_{y} & \dot{v}_{y}\end{array}\right]^{\mathrm{T}}$ then the EKF predict-update equations can be formulated using the absolute velocity measurements as a-posteriori corrections [32, 33]. It should be noted that the Jacobians used through the EKF implementation were based on the linearised planar dynamics as found in [27].

Finally, by integration, the absolute $x, y$, position expressed in EFF can be estimated for a quadrotor flying indoors. The overall position fusion scheme is presented in Fig. 3, where the interface, between the Tam 2 vision chip and the I2A optic-flow algorithm have been implemented using an AVR ATmega 328P processor. The opticflow measurements are being updated every $30 \mathrm{~ms}$ which indicates the low computational power required for this optic-flow solution.

\section{Switching model predictive control}

The design of the proposed SMPC-scheme is based on three cascade switching model predictive controllers applied on: (a) the multiple PWA representations of the attitude dynamics subsystem, and (b) on the error dynamics modelling of the quadrotor's vertical and horizontal motions respectively. The overall block diagram of the closed loop system is depicted in Fig. 4.

The position control generates control commands that act as reference inputs for the attitude controller. For slow position deviations, the control actions $u_{x}, u_{y}$ can be approximated with $\theta^{r},-\phi^{r}$ respectively, if the yaw angle $\psi$ is constantly commanded to remain zero. Subsequently, $-\phi^{r}, \theta^{r}, \psi^{r}=0$, their rates and their integrals over time are passed as references to the attitude controller. The most demanding part of the control design process is that of attitude control which, must be able to accurately track the rapidly changing reference angles.

The construction of the SMPCs for each of the vertical, horizontal and rotary motion $\left(E_{z} \rightarrow x_{E_{z}}, E_{x} E_{y} \rightarrow\right.$ $\left.x_{E_{x} E_{y}}, \eta \rightarrow x_{\eta}\right)$ subsystems, follows the same methodology. Considering two distinct $T_{\mathrm{s}}^{\eta}, T_{\mathrm{s}}^{t}$ sampling periods for the 


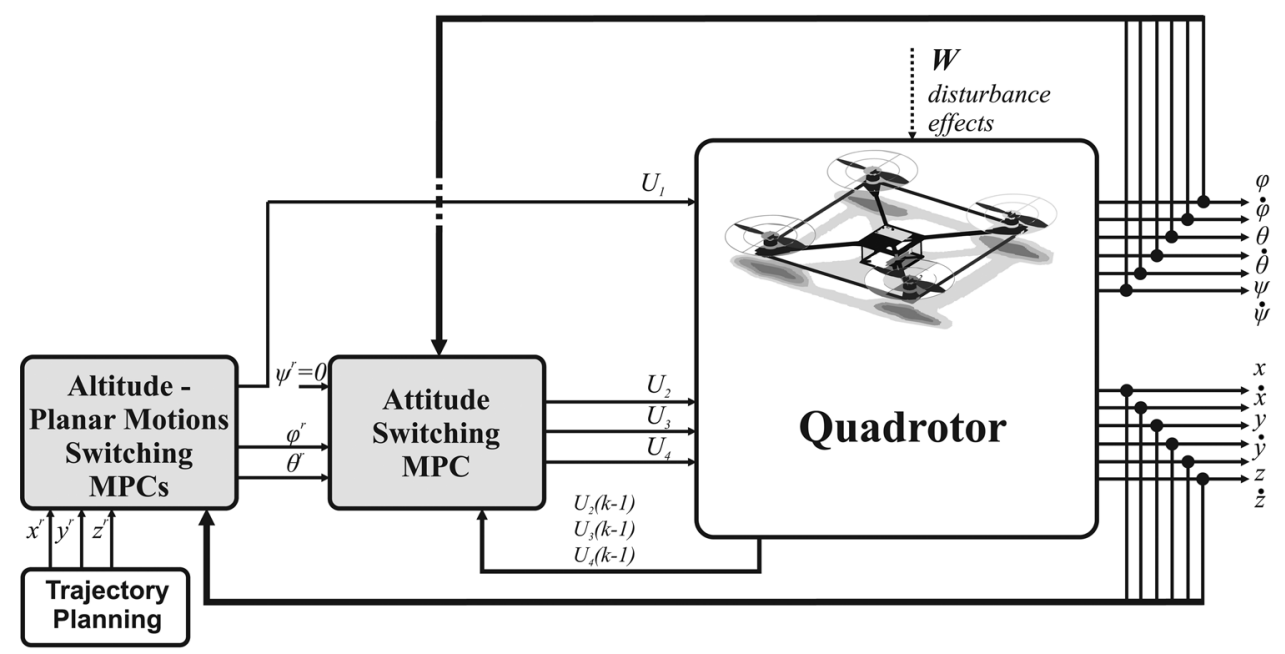

Fig. 4 Switching 6-DOF MPC scheme

attitude and vertical-horizontal dynamics, respectively, all described equations for vertical, horizontal and attitude dynamics equation are expressed as discrete time PWA systems

$$
\mathbf{x}_{\ell}(k+1)=\overline{\mathbf{A}}_{\ell}^{j} \mathbf{x}_{\ell}(k)+\overline{\mathbf{B}}_{\ell}^{j} \mathbf{u}_{\ell}(k)+\tilde{\mathbf{w}}_{\ell}
$$

where $\ell$ is the index for each subsystem $\ell \in\left\{E_{z}, E_{x} E_{y}, \eta\right\}$, and $\mathbf{x}_{\ell}(k) \in \mathcal{X}^{\ell} \subseteq \mathfrak{R}^{\ell}$ is the discrete state vector of each system, $\mathbf{u}_{\ell}(k) \in \mathcal{U}^{\ell} \subseteq \mathfrak{R}^{\ell}, u_{l} \in\left\{u_{E_{z}}, u_{E_{x} E_{y}}, u_{\eta}\right\}$ is the corresponding control action at the discrete time instant $k, \overline{\mathbf{A}}_{\ell}^{\mathbf{j}}, \overline{\mathbf{B}}_{\ell}^{\mathbf{j}}$ are the discretised corresponding state space matrices for the horizontal, vertical and rotational motions of the quadrotor, respectively, and $\tilde{\mathbf{w}}_{\ell}$ term corresponds to the effect of the unknown additive disturbances on the system's dynamics. Moreover, $j \in \mathcal{S}$ with $\mathcal{S} \triangleq$ $\left\{1,2, \ldots, s^{\ell}\right\}$, is a finite set of indexes and $s^{\ell}$ denotes the number of PWA subsystems in (16) for the $\ell$ th subsystem. For polytopic uncertainty, let $\Sigma^{\ell}$ be the polytope

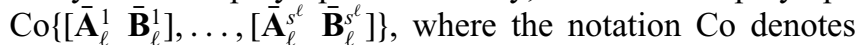
the convex hull of the set defined by its vertices $\left[\overline{\mathbf{A}}_{\ell}^{j}, \overline{\mathbf{B}}_{\ell}^{j}\right]$. Any $\left[\overline{\mathbf{A}}_{\ell}^{j}, \overline{\mathbf{B}}_{\ell}^{j}\right]$ within the convex set $\Sigma^{\ell}$, is a linear combination of

$$
\left[\overline{\mathbf{A}}_{\ell}^{j}, \overline{\mathbf{B}}_{\ell}^{j}\right]=\sum_{j=1}^{s^{\ell}} a_{j}\left[\overline{\mathbf{A}}_{\ell}^{j}, \overline{\mathbf{B}}_{\ell}^{j}\right], \quad \sum_{j=1}^{s^{\ell}} a_{j}=1, \quad 0 \leq a_{j} \leq 1
$$

The sets $\mathcal{X}^{\ell}$ and $\mathcal{U}^{\ell}$ specify state and input constraints and it is assumed they are compact polyhedral sets.

Generally, the state and input constraints should be set in relation to the application profile. For simplicity, the specification of the constraints is achieved under the assumption that the origin is an equilibrium state, with $\mathbf{u}^{\ell}(0)=\mathbf{0}$. For the $j$ th linearised subsystem, let the set $\mathcal{X}_{j}^{\ell}$ contain the $\mathbf{x}_{\ell, l}^{\circ, j}$ states that satisfy the following inequality

$$
\mathbf{x}_{\ell, l}^{\circ, j} \in \mathcal{X}_{j}^{\ell}: \mathbf{x}_{\ell, l}^{\circ, j, \min }=\mathbf{x}_{\ell, l}^{\circ, j}-\Delta_{\ell, \iota} \leq \mathbf{x}_{\ell, l}^{\circ, j} \leq \mathbf{x}_{\ell, l}^{\circ, j}+\Delta_{\ell, \iota}=\mathbf{x}_{\ell, l}^{\circ, j \max }
$$

where the subscript index corresponds to the $i$ th component of the $\mathbf{x}$-vector (i.e. $\iota=2, \ell \rightarrow x_{\eta}$ corresponds to the $\dot{\phi}^{\circ, j}$ variable), $\Delta_{\ell, \iota}>0$ and $\mathcal{X}=\bigcup \mathcal{X}_{i}^{\ell}, \iota=1, \ldots, m$, where $m$ denotes the length of $x_{\ell}^{\circ, j}$. For the SMPC-synthesis, the following state constraints have been used.

$$
\left[\begin{array}{c}
-\frac{\pi}{4} \\
-\pi \\
-0.75 \\
-1 \\
-3
\end{array}\right]<\left[\begin{array}{c}
\phi, \theta \\
\psi \\
\dot{\phi}, \dot{\theta} \\
\dot{\psi} \\
\dot{z}, \dot{x}, \dot{y}
\end{array}\right]<\left[\begin{array}{c}
\pi \\
4 \\
\pi \\
0.75 \\
1 \\
3
\end{array}\right]
$$

The control inputs bounding sets $\mathcal{U}^{\ell}$ can be derived from the bounds on the motors' angular velocities $\Omega_{\kappa}, \kappa=1, \ldots, 4$, $\Omega_{\kappa} \in\left[0, \Omega_{\kappa}^{\max }\right]$ by using interval analysis [34]. Particularly, the constraints on the control inputs are formulated as (see equation at the bottom of the page)

The constraints on the rate of change of the control action are related to the time constant of the speed controller-motor-propeller system and affects the transient performance of the quadrotor. The constraints of the rate of the control actions are modelled as $\Delta U_{i}^{\min } \leq \Delta U_{i} \leq$ $\Delta U_{i}^{\max }, i=1,2,3,4, \Delta \Omega_{\mathrm{r}}^{\min } \leq \Delta \Omega_{\mathrm{r}} \leq \Delta \Omega_{\mathrm{r}}^{\max }$. All state and input constraints have been combined by using a set of $\mathbf{H}_{\mathbf{i}}^{l}$ zeroed $2 \times(m+n)$ matrices except for their $i$ th column, which is equal to $[1,-1]^{\mathrm{T}}$, where $m$ is the number of states of vector $x_{\ell}$ and $n$ the number of control actions $u_{\ell}$. Specifically, for each of the individual subsystems over which the controller is constructed, the constraints are formulated as follows

$$
\begin{array}{lccl}
\delta U_{1}^{\min } & & 0 \leq \delta U_{1} \leq b \sum_{i=1}^{4}\left(\Omega_{1}^{\max }\right)^{2} & =\delta U_{1}^{\max } \\
\delta U_{2}^{\min } & = & -b\left(\Omega_{2}^{\max }\right)^{2} \leq \delta U_{2} \leq b\left(\Omega_{4}^{\max }\right)^{2} & =\delta U_{2}^{\max } \\
\delta U_{3}^{\min } & = & -b\left(\Omega_{3}^{\max }\right)^{2} \leq \delta U_{3} \leq b\left(\Omega_{1}^{\max }\right)^{2} & =\delta U_{3}^{\max } \\
\delta U_{4}^{\min } & = & -d\left[\left(\Omega_{1}^{\max }\right)^{2}+\left(\Omega_{3}^{\max }\right)^{2}\right] \leq \delta U_{4} \leq d\left[\left(\Omega_{2}^{\max }\right)^{2}+\left(\Omega_{4}^{\max }\right)^{2}\right] & =\delta U_{4}^{\max } \\
\delta \Omega_{\mathrm{r}}^{\min } & & -\Omega_{1}^{\max }-\Omega_{3}^{\max } \leq \delta \Omega_{\mathrm{r}} \leq \Omega_{2}^{\max }+\Omega_{4}^{\max } & =\delta \Omega_{\mathrm{r}}^{\max }
\end{array}
$$


Vertical motion subsystem

$$
\left[\begin{array}{c}
\mathbf{H}_{1}^{E_{z}} \\
\mathbf{H}_{2}^{E_{z}} \\
\mathbf{H}_{3}^{E_{z}} \\
\mathbf{H}_{4}^{E_{z}} \\
\mathbf{H}_{5}^{E_{z}}
\end{array}\right]_{10 \times 5} \cdot\left[\begin{array}{c}
\mathbf{x}_{E_{z}} \\
\mathbf{u}_{E_{z}}
\end{array}\right]_{5 \times 1} \leq\left[\begin{array}{c}
x_{E_{z}, 1}^{\circ, j, \max } \\
x_{E_{z}, 1}^{\circ, \min } \\
x_{E_{z}, 2}^{\circ, \max } \\
x_{E_{z}, 2}^{\circ, \min } \\
x_{E_{z}, 3}^{\circ,, \max } \\
x_{E_{z}, 3}^{\circ, \min } \\
\overline{U_{1}^{\max }} \\
\delta U_{1}^{\min } \\
0 \\
\Delta U_{1}^{\max }
\end{array}\right]
$$

Horizontal motion subsystem

$$
\left[\begin{array}{c}
\mathbf{H}_{1}^{E_{x} E_{y}} \\
\vdots \\
\mathbf{H}_{6}^{E_{x} E_{y}} \\
-\mathbf{H}_{7}^{E_{x} E_{y}} \\
\mathbf{H}_{8} E_{x} E_{y}
\end{array}\right]_{16 \times 8} \cdot\left[\begin{array}{c}
\mathbf{x}_{\xi}^{E_{x} E_{y}} \\
- \\
\mathbf{u}_{E_{x} E_{y}}
\end{array}\right]_{8 \times 1} \leq\left[\begin{array}{c}
x_{E_{x} E_{y}, 1}, \max \\
x_{E_{x} E_{y}, 1}^{\circ, j i n} \\
\vdots \\
x_{E_{x} E_{y}, 6}^{\circ, \max } \\
x_{E_{x} E_{y}, 6} \\
- \\
\delta u_{x}^{\max } \\
\delta u_{x}^{\min } \\
\delta u_{y}^{\max } \\
\delta u_{y}^{\min }
\end{array}\right]
$$

Attitude subsystem

$$
\left[\begin{array}{c}
\mathbf{H}_{1}^{\eta} \\
\vdots \\
\mathbf{H}_{9}^{\eta} \\
\overline{\mathbf{H}_{10}^{\eta}} \\
\vdots \\
\mathbf{H}_{14}^{\eta} \\
\mathbf{H}_{15}^{\eta} \\
\vdots \\
\mathbf{H}_{19}^{\eta}
\end{array}\right]_{38 \times 19} \cdot\left[\begin{array}{c}
\mathbf{x}_{\eta}^{\circ, j, \max } \\
\overline{\mathbf{u}^{\eta}}
\end{array}\right]_{19 \times 1} \leq\left[\begin{array}{c}
x_{\eta, 1}^{\circ, j, \min } \\
x_{\eta, 9}^{\circ, j, \max } \\
x_{\eta, 9}^{\circ, j, \min } \\
\delta \overline{U_{1}^{\max }} \\
\delta U_{1}^{\min } \\
\vdots \\
\delta \Omega_{\mathrm{r}}^{\max } \\
\delta \Omega_{\mathrm{r}}^{\min } \\
0 \\
\Delta U_{1}^{\max } \\
\vdots \\
0 \\
\Delta \Omega_{\mathrm{r}}^{\max }
\end{array}\right]
$$

These constraints are embedded in the SMPC calculation algorithm by using the dual optimisation method. The $\tilde{\mathbf{w}}_{\ell}$ term corresponding to the effect of the unknown additive disturbances on the system's dynamics can be analysed in general as $\tilde{\mathbf{w}}_{\ell}=\mathbf{B}_{d}^{\ell} \mathbf{d}^{\ell}(\mathbf{k})$, where $\mathbf{d}^{\ell}(k)$ is the output of the following linear system

$$
\begin{array}{r}
\mathbf{x}_{d}^{\ell}(k+1)=\hat{\mathbf{A}}_{\ell} \mathbf{x}_{d}^{\ell}(k)+\hat{\mathbf{B}}_{\ell} \mathbf{n}_{d}^{\ell}(k) \\
\mathbf{d}^{\ell}(k)=\hat{\mathbf{C}}_{\ell} \mathbf{x}_{d}^{\ell}(k)+\hat{\mathbf{D}}_{\ell} \mathbf{n}_{d}^{\ell}(k)
\end{array}
$$

The system described in (23) is driven by random Gaussian noise $\mathbf{n}_{d}^{\ell}(k)$, having zero mean and unit covariance matrix, while it should be mentioned that in classical quadrotormodelling this term is missing, despite its natural inclusion. In the work presented in this paper, the bounds for the disturbance $\mathbf{d}^{\ell}$ have being experimentally measured by applying forcible gusts and measuring their maximum effect on the quadrotor's attitude.

Since the vector $\mathbf{x}_{d}^{\ell}(k)$ is not directly measurable, its prediction $\hat{\mathbf{x}}_{d}^{\ell}(k)$ is obtained based on an extended Kalman state estimator, based on (16), (23) and the corresponding notation of $\tilde{\mathbf{w}}_{\ell}$

$$
\begin{aligned}
{\left[\begin{array}{c}
\mathbf{x}^{\ell}(k+1) \\
\hat{\mathbf{x}}_{d}^{\ell}(k+1)
\end{array}\right]=} & {\left[\begin{array}{cc}
\overline{\mathbf{A}}_{\ell}^{j} & \mathbf{B}_{\mathbf{d}}^{\ell} \hat{C}_{\ell} \\
0 & \hat{\mathbf{A}}_{\ell}
\end{array}\right]\left[\begin{array}{c}
\mathbf{x}_{\ell}(k) \\
\hat{\mathbf{x}}_{d}^{\ell}(k)
\end{array}\right]+\left[\begin{array}{c}
\overline{\mathbf{B}}_{\ell}^{j} \\
0
\end{array}\right] \mathbf{u}_{\ell}(k) } \\
& +\left[\begin{array}{c}
\mathbf{B}_{\mathbf{d}}^{\ell} \hat{\mathbf{D}}_{\ell} \\
\hat{\mathbf{B}}_{\ell}
\end{array}\right] \mathbf{n}_{\mathbf{d}}^{\ell}(k)
\end{aligned}
$$

It must be noted that currently there is an important research work going on in the area of disturbance observation which increases the capabilities of the feedback controller as in [35].

The basic idea of SMPC is to calculate a sequence of future control actions in a way that will minimise a cost function defined over a predefined prediction horizon. The cost to be optimised is the expectation of a quadratic function, measuring the distance between the predicted system's output and some predicted reference sequence, over the control horizon $N_{\mathrm{c}}$, and a quadratic function measuring the control effort. More specifically, the $(\ell, j)$ th SMPC's objective is to optimise the quadratic cost in (26), whereas the $(\ell, j)$ th discrete linearised system is within $\Sigma^{\ell}$. The tuning of prediction $N_{\mathrm{p}}$ and control $N_{\mathrm{c}}$ horizons is a coupled process. Additionally, the response of the system can also be shaped using weighting matrices, applied on the system outputs, the control action and the control rates respectively. Each MPC $V_{\text {MPC }}^{\ell, j}(k)$, corresponding to the $\ell, j$ th PWA model of the quadrotor's motion at time $k$, is being obtained by solving the following optimisation problem, defined by the cost

$$
\min _{\Delta u_{\ell}} J(k)
$$

subject to the $\ell, j$ th PWA system dynamics and their associated constraints, where $J(k)$ is (see (26))

where $\ell \rightarrow x_{\eta}, x_{E_{x} E_{y}}, x_{E_{z}}$ indicates that all parameters refer either to the attitude, altitude or horizontal motion PWA

$$
\begin{aligned}
J(k)= & \sum_{i=N_{w}^{\ell}}^{N_{p}^{\ell}}\left[\hat{\mathbf{x}}_{\ell}(k+i \mid k)-\mathbf{r}^{\ell}(k+i \mid k)\right]^{\mathrm{T}} \mathbf{Q}^{\ell}\left[\hat{\mathbf{x}}_{\ell}(k+i \mid k)-\mathbf{r}^{\ell}(k+i \mid k)\right]+\sum_{i=0}^{N_{c}^{\ell}-1}\left[\Delta \mathbf{u}_{\ell}^{\mathrm{T}}(k+i \mid k) \mathbf{R}^{\ell} \Delta \mathbf{u}_{\ell}(k+i \mid k)\right] \\
& +\sum_{i=N_{w}^{\ell}}^{N_{p}^{\ell}}\left[\mathbf{u}_{\ell}(k+i \mid k)-\mathbf{s}^{\ell}(k+i \mid k)\right]^{\mathrm{T}} \mathbf{N}^{\ell}\left[\mathbf{u}_{\ell}(k+i \mid k)-\mathbf{t}^{\ell}(k+i \mid k)\right]
\end{aligned}
$$




\section{www.ietdl.org}

dynamics, $i$ is the index along the prediction horizon, $N_{\mathrm{w}}^{\ell}$ is the beginning of the prediction horizon, $\mathbf{Q}^{\ell}$ is the state error weight matrix, $\mathbf{R}^{\ell}$ is the rate of change in control weight matrix, $\mathbf{N}^{\ell}$ is the control action error weight matrix, $\hat{\mathbf{x}}_{\ell}(k+$ $i \mid k)$ is the predicted attitude, altitude or horizontal translation system state vector at time $k+i, \mathbf{r}^{\ell}(k+i \mid k)$ is the setpoint profile at time $k+i, \Delta \mathbf{u}_{\ell}(k+i \mid k)$ is the predicted rate of change in control action at time $k+i, \mathbf{u}_{\ell}(k+i \mid k)$ is the predicted optimal control action at time $k+i$, and $\mathbf{t}^{\ell}(k+i \mid k)$ is the input set-point profile at time $k+i$. This computation problem is solved for attitude, altitude and horizontal motion dynamics. At each moment the three attitude, altitude and horizontal motions controllers $V_{\mathrm{MPC}}^{\ell, j}(k)$ are computed over the selected PWA system in a switching manner according to the current regime of the estimated state vector $\mathbf{x}_{\ell}$. It should be noted that if $s^{\ell}$ increases, then the approximation of the nonlinear system by a large number of linearised systems is more accurate and results in a larger flight envelope. Note also that the altitude and planar motion's set-point profiles are being provided explicitly in order to fit the required path planning commands, where the attitude control set-point profiles are being provided from the output of the planar motion's controller, as the $N_{\mathrm{c}}$ number of predictions of its control actions, which turn to be the reference commands for the attitude controller.

Under the assumption of $(\ell, s)$ discrete PWA systems and $(\ell, s)$ available $V_{\mathrm{MPC}}^{\ell, j}$ controllers, the objective of the $j \in \mathcal{S}^{\ell}$ controller is to stabilise the $(\ell, j)$ th system. If $s$ increases, then the approximation of the nonlinear system by a large number of PWA systems is more accurate for a larger part of the flight envelope, enabling the development of more efficient flight control algorithms. Although, the computation of multiple model predictive controllers over a family of PWA systems cannot guarantee the stability of the nonlinear system during switching, through careful selection of the linearisation points, no stability issues were observed in all the experimental test-cases.

The switching between the different controllers is ruled by the values of the state vector and the utilised family of PWA representations. Assuming that $(\ell, s)$ discrete PWA systems are used, then the operation points for which each one of these PWA systems was calculated define the regions that each PWA is active. Specifically, the $\ell \rightarrow x_{\eta}, x_{E_{x} E_{y}}, x_{E_{z}}$ attitude, position or altitude control scheme switches to the $V_{\mathrm{MPC}}^{\ell, j}$ controller according to the following formula

$$
\underset{(\ell, j)}{\operatorname{argmin}}|| \mathbf{x}_{\ell}-\mathbf{x}_{\ell, j}^{\circ} \|
$$

where $\|\cdot\|$ represents the Euclidean norm in vector form.

Regarding the set-point profile generation, it should be clarified that in the ensuing experimental test-cases the translational position profile was explicitly provided as a reference trajectory. On the other hand, the set-point profile of the attitude controller is provided by the output commands of the position control loops.

\section{Experimental studies}

\subsection{Experimental set-up}

The UPATcopter is equipped with an advanced main control unit Kontron ${ }^{\mathrm{TM}}$ pITX single board computer (SBC) comprising of an ATOM Z530 1.6 GHz CPU with $2 \mathrm{~GB}$ of RAM, a $150 \mathrm{~GB}$ solid state disk drive and the sensor

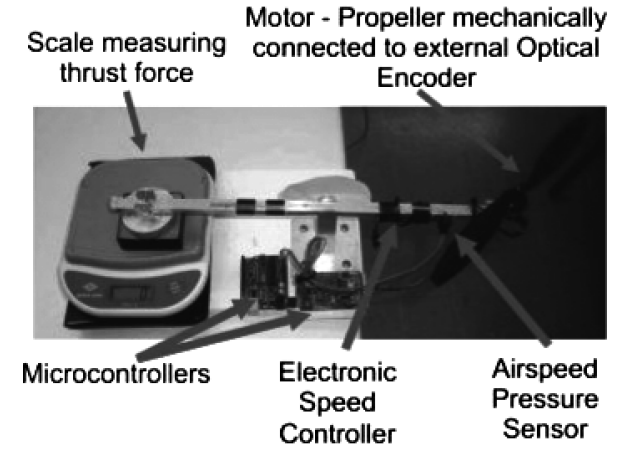

Fig. 5 Experimental set-up used for motor-propeller characteristics measurements

system described in Section 3. The utilisation of this unit offers several advantages including: (a) the ability to deploy sophisticated control laws programmed in highlevel languages, (b) the ability to seamlessly use several off-the-shelf sensors, (c) the ability to connect to urban wireless networks and exchange data with ground stations or other unmanned systems in the area, and (d) the ability to perform complex computations including advanced image processing. The JIDA32 interface of the pITX SBC is used to communicate with the brushless electronic speed controllers (ESCs) via the $\mathrm{I} 2 \mathrm{C}$ protocol while concurrently providing sampled data from a Maxbotix EZ1 Sonarbased altitude data to the SBC. The utilised Maxbotix EZ1 Sonar with a $30^{\circ}$ beam angle manages to measure altitude despite the quadrotor's attitude deviations but the selection of a wide beam could not reject the ambient noise. The utilised Robbe ${ }^{\mathrm{TM}} 2827-35$ brushless motors have high-thrust characteristics and lift the prototype quadrotor at a level lower than the $50 \%$ of their total thrust. An experimental set-up was developed in order to measure the motor-propeller characteristics. Specifically, the developed set-up, shown in Fig. 5, provided measurements of the output thrust, rotor rate of rotation, current feedback at the dcbrushless motor and produced airflow owing to the rotation of propeller.

In Fig. 6, the power efficiency the response of the BLCTRL V2.0 ESC-motor/propeller system is presented, where the motors could lift a maximum weight of over $3 \mathrm{~kg}$. The developed quadrotor weighs $1.1 \mathrm{~kg}$ with a $3300 \mathrm{mAh}$ 3 Cell Vislero ${ }^{\mathrm{TM}}$ battery and achieves $12 \mathrm{~min}$ of autonomous hovering flight. The quadrotor prototype is equipped with a Wi-Fi 802.11n adaptor which is used both for telemetry and communication with other systems.

Fig. 7 explains the main hardware diagram of the quadrotor prototype. The quadrotor's variables are listed in Table 2. Based on the listed values, the following constraints on the inputs where $0 \leq U_{1} \leq 36.63,\left|U_{2}\right| \leq 18.319,\left|U_{3}\right| \leq$ $18.319,\left|U_{4}\right| \leq 0.22$.

The tuning parameters of the SMPC were $\mathbf{R}_{\mathbf{u}_{\eta}}^{\eta}=20$. $\mathbf{I}_{5}, \quad \mathbf{R}_{\Delta \mathbf{u}_{\mathrm{j}}^{\eta}, j}^{\eta}=200 \cdot \mathbf{I}_{5}, \mathbf{Q}^{\eta}=20^{4} \cdot \operatorname{diag}(1,2,1,1,2,1,1,2,1)$, for all $j$ attitude PWA-utilised subsystems, $\mathbf{R}_{\mathbf{u}_{\mathbf{j}}^{\mathbf{E}_{\mathbf{z}}}, j}^{E_{z}}=10$, $\mathbf{R}_{\Delta \mathbf{u}_{\mathbf{j}}^{\mathbf{E}_{\mathbf{z}}, j}}^{\mathbf{E}_{\mathbf{z}}}=100, \mathbf{Q}^{\mathbf{E}_{\mathbf{z}}}=10^{4} \cdot \mathbf{I}_{3}$, for all $v$ altitude PWAutilised subsystems, and $\mathbf{R}_{\mathbf{u}_{\mathbf{E}} \mathbf{E}_{\mathbf{x}}}^{E_{x} E_{y}}=10 \cdot \mathbf{I}_{2}, \mathbf{R}_{\Delta \mathbf{u}_{\mathbf{j}}}^{\mathbf{E}_{\mathbf{y}} \mathbf{E}_{\mathbf{y}}, j}=100 \cdot$ $\mathbf{I}_{2}, \mathbf{Q}^{\mathbf{E}_{\mathbf{x}} \mathbf{E}_{\mathbf{y}}}=10^{2} \cdot \mathbf{I}_{6}$, for all $p$ horizontal motions PWAutilised subsystems. The prediction and control horizon for all subsystems were set to $N_{\mathrm{p}}=5$ and $N_{\mathrm{c}}=2$, respectively. It should be noted that the position controller feeds the first 

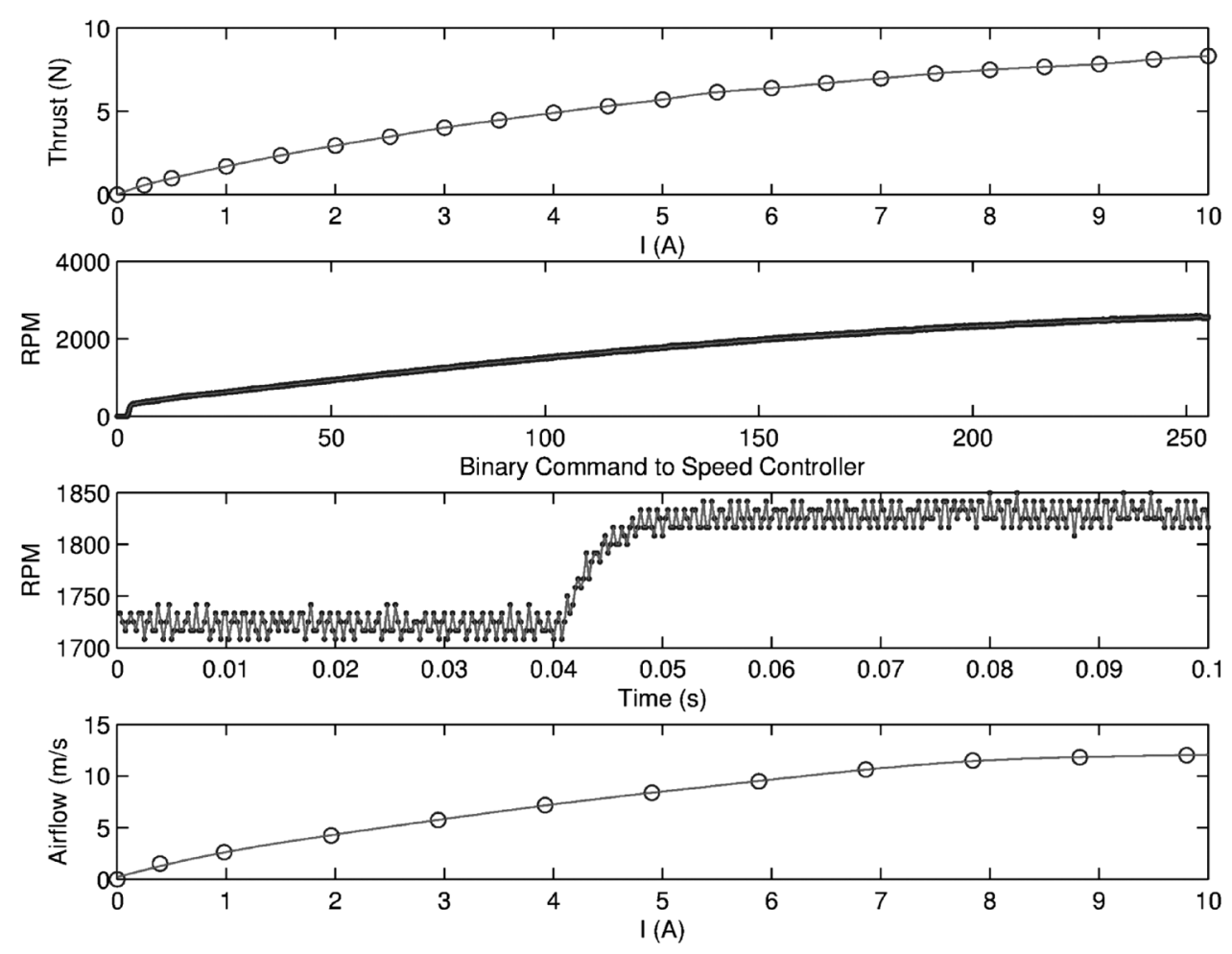

Fig. 6 Experimentally measured current-thrust, RPM-binary command to speed controller, RPM step response, airflow-current curves

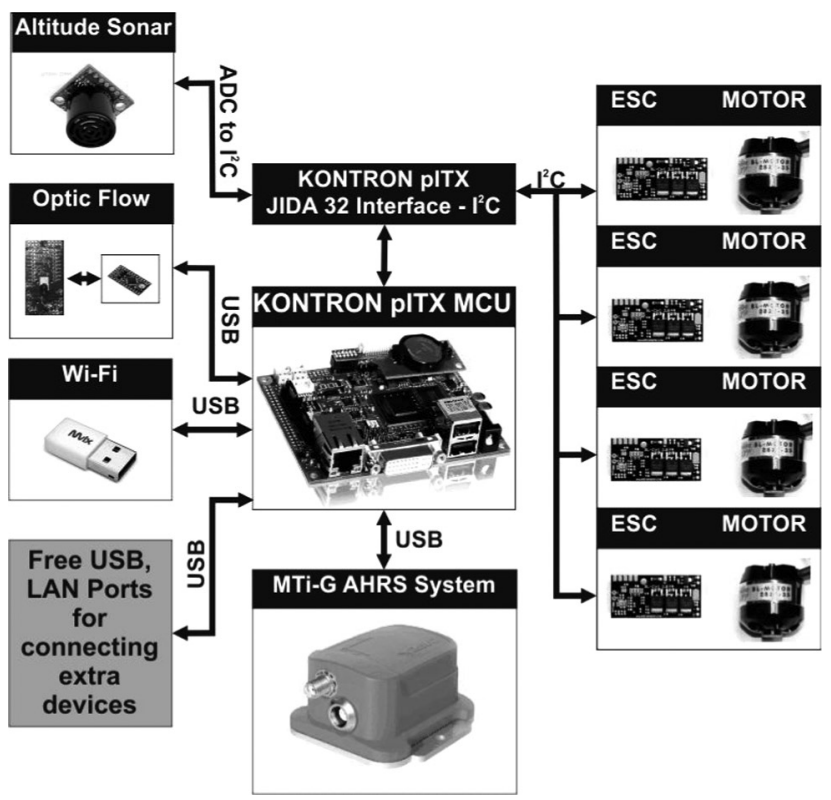

Fig. 7 UPATcopter main hardware diagram

Table 2 Quadrotor model parameters

\begin{tabular}{lll}
\hline Design variable & \multicolumn{1}{c}{ Value } & Units \\
\hline$m_{\mathrm{s}}$ & 1.1 & $\mathrm{~kg}$ \\
$l_{\mathrm{a}}$ & 0.21 & $\mathrm{~m}$ \\
$I_{x x}=I_{y y}$ & 0.0196 & $\mathrm{~kg} \mathrm{~m}^{2}$ \\
$I_{z z}$ & 0.0264 & $\mathrm{~kg} \mathrm{~m}^{2}$ \\
$J_{\mathrm{r}}$ & $8.5 \times 10^{-4}$ & $\mathrm{~kg} \mathrm{~m}^{2}$ \\
$b$ & $9.29 \times 10^{-5}$ & $\mathrm{~N} \mathrm{~s}^{2}$ \\
$d$ & $1.1 \times 10^{-6}$ & $\mathrm{~N} \mathrm{~m} \mathrm{~s}^{2}$ \\
\hline
\end{tabular}

two ( since $N_{\mathrm{c}}=2$ ) elements of the set-point profile vector of the attitude controller, where the rest three elements repeat the last value provided by position controller. The robust behaviour to disturbances has been calculated for

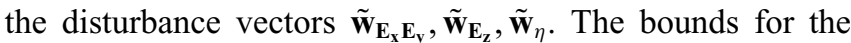
additive disturbances under induced wind conditions were experimentally measured and were set as $\mathbf{d}^{\eta}=\left|\tilde{\mathbf{w}}_{\eta, \max }\right|=$ $[0,0.15 \mathrm{rad} / \mathrm{s}, 0,0,0.15 \mathrm{rad} / \mathrm{s}, 0,0,0.15 \mathrm{rad} / \mathrm{s}, 0]^{\mathrm{T}}, \quad \mathbf{d}^{E_{z}}=$ $\left|\tilde{\mathbf{w}}_{\mathbf{E}_{\mathbf{z}}, \max }\right|=[0,0.1 \mathrm{~m} / \mathrm{s}, 0]^{\mathrm{T}}, \mathbf{d}^{E_{x} E_{y}}=\left|\tilde{\mathbf{w}}_{\mathbf{E}_{\mathbf{x}} \mathbf{E}_{\mathbf{y}}, \max }\right|=[0,0.1 \mathrm{~m} / \mathrm{s}$, $0,0,0.1 \mathrm{~m} / \mathrm{s}, 0]^{\mathrm{T}}$. Based on the measurements presented in Fig. 6, the rate of change of the control actions were constrained to $\pm 40 \%$ of the maximum value of respective control laws.

For the design and experimental application of the SMPC scheme $s=9$ attitude PWA-systems, and $s=2$ altitude PWA-systems, together with the linear model of the horizontal motions, have been utilised. In all these cases, the sampling period was set to $T_{\mathrm{s}}^{\eta}=0.0083 \mathrm{~s}(120 \mathrm{~Hz})$ for the attitude controller which is equal to the maximum Xsens MTi-G IMU update rate and $T_{\mathrm{s}}^{t}=0.03 \mathrm{~s}(33 \mathrm{~Hz})$ for the altitude and horizontal motions controllers. It should be noted that the spectral separation between the attitude and translational controllers is needed in order to avoid conflicts between the two loops [27]. The PWA regions because of the used linearisation points can be found based on the parameters listed in Table 3.

\subsection{Flight results}

In order to justify the efficiency of the proposed control approach, various flight test-cases have been performed including: (a) position hold and altitude set-point, (b) trajectory tracking, (c) hovering and (d) aggressive attitude control.

In the first case, the overall control scheme managed to effectively hold the quadrotor's horizontal position in a circle of radius generally less than $0.15 \mathrm{~m}$ while also 
Table 3 PWA operation regions and linearisation points $(\Gamma \gg 1)$

\begin{tabular}{lccc}
\hline PWA & Region min & Linearisation point & Region max \\
\hline 1 & -0.5 & $\dot{\phi}^{\circ, j}=\dot{\theta}^{\circ, j}=0(\mathrm{rad} / \mathrm{s})$ & 0.5 \\
2 & 0.5 & $\dot{\phi}^{\circ, j}=1(\mathrm{rad} / \mathrm{s})$ & $\Gamma$ \\
3 & $-\Gamma$ & $\dot{\phi}^{\circ, j}=-1(\mathrm{rad} / \mathrm{s})$ & -0.5 \\
4 & 0.5 & $\dot{\theta}^{\circ} j=1(\mathrm{rad} / \mathrm{s})$ & $\Gamma$ \\
5 & $-\Gamma$ & $\dot{\theta}^{\circ, j}=-1(\mathrm{rad} / \mathrm{s})$ & -0.5 \\
6 & 0.5 & $\dot{\phi}^{\circ, j}$ and $\dot{\theta}^{\circ, j}=1(\mathrm{rad} / \mathrm{s})$ & $\Gamma$ \\
7 & $-\Gamma$ & $\dot{\phi}^{\circ, j}, \dot{\theta}^{\circ, j}=-1(\mathrm{rad} / \mathrm{s})$ & -0.5 \\
8 & $0.5,-\Gamma$ & $\dot{\phi}^{\circ, j}=1, \dot{\theta}^{\circ, j}=-1(\mathrm{rad} / \mathrm{s})$ & $\Gamma,-0.5$ \\
9 & $0.5,-\Gamma$ & $\dot{\phi}^{\circ, j}=-1, \dot{\theta}^{\circ, j}=1(\mathrm{rad} / \mathrm{s})$ & $-0.5, \Gamma$ \\
1 & -0.1 & $\theta, \phi(\mathrm{rad})$ & 0.15 \\
2 & \multicolumn{4}{c}{0.15} \\
1 & \multicolumn{4}{c}{ or $|\theta|>(\mathrm{rad})$} \\
\hline
\end{tabular}

achieving altitude control, a performance that is better of the one achieved from an experienced radio control (RC)pilot [12] and comparable with the results reported in [12, 36]. The results for a height altitude reference equal to $1.5 \mathrm{~m}$ for the first $10 \mathrm{~s}$ of the flight and $2 \mathrm{~m}$ for the remaining $10 \mathrm{~s}$ are shown in Fig. 8, and in Fig. 9 the horizontal position combined with the optic-flow deviation measurements and the altitude measurements are presented.

The same position hold and altitude set-point control test-cases were examined under presence of a $x(4.96 \mathrm{~m} / \mathrm{s})$, $y(1.31 \mathrm{~m} / \mathrm{s})$ and $z(1.22 \mathrm{~m} / \mathrm{s})$ directional wind gust. The wind gust is only present in a segment of the quadrotor's path and specifically after the quadrotor achieves an altitude higher than $1.8 \mathrm{~m}$ (reached at $12.7 \mathrm{~s}$ ). The wind gusts are produced using electric turbines and a multiple pipe tunnel. The wind gusts exit the tunnel having a laminar flow characteristic; the wind-gust generating set-up is presented in Fig. 10.

The corresponding 3D experimental measured flight path of the quadrotor's flight in order to track the desired altitude reference and hold position under presence of a $x(4.96 \mathrm{~m} / \mathrm{s}), y(1.31 \mathrm{~m} / \mathrm{s})$ and $z(1.22 \mathrm{~m} / \mathrm{s})$ directional wind gust indicated in Fig. 11 where the horizontal positions combined with the optic-flow deviation measurements and the altitude measurements are similarly presented in Fig. 12. The maximum deviation $x$-axis is less than $0.20 \mathrm{~m}$ and less than $0.1 \mathrm{~m}$ in the $y$-axis.

The small error in all $E_{x} E_{y} E_{z}$-axis indicate the overall efficiency of the controller, which is notable since the quadrotor faces complex aerodynamic phenomena when an airflow disturbs the propellers, including total thrust variation and blade flapping [37]. Assuming totally laminar flow then following the analysis presented in [37] that (a) blade flapping is caused because of the fact that when a relative airflow disrupts the propellers' motion the advancing blade has a higher velocity relative to the air, whereas the retreating blade has a lower velocity which in fact leads to lift variation, and (b) thrust variation is caused because of the fact that the total airflow has a different velocity than the free stream speed. However, these effects become even more complex when the wind gust presents turbulence. An analysis on rotorcraft response subject to atmospheric turbulence is presented in [22].

The overall system was also tested in trajectory tracking by commanding the quadrotor to follow a straight line in $E_{y}$-axis and hold its position once it has reached the desired set-point. The corresponding results are presented in Figs. 13 and 14. Despite the limitations induced by the mere existence of only relevant measurements of the quadrotor's displacement from the optic-flow sensor, the proposed control scheme managed to effectively track the desired reference path.

In order to measure the controller's efficiency the MSEs of the measured responses are presented in Fig. 15. As it is clearly being presented, the overall control structure efficiently minimises the error between the quadrotor's response and the reference trajectory even in the case of forcible wind gusts. The relatively high MSE in the $E_{y}$-axis response for the case of trajectory following is being related

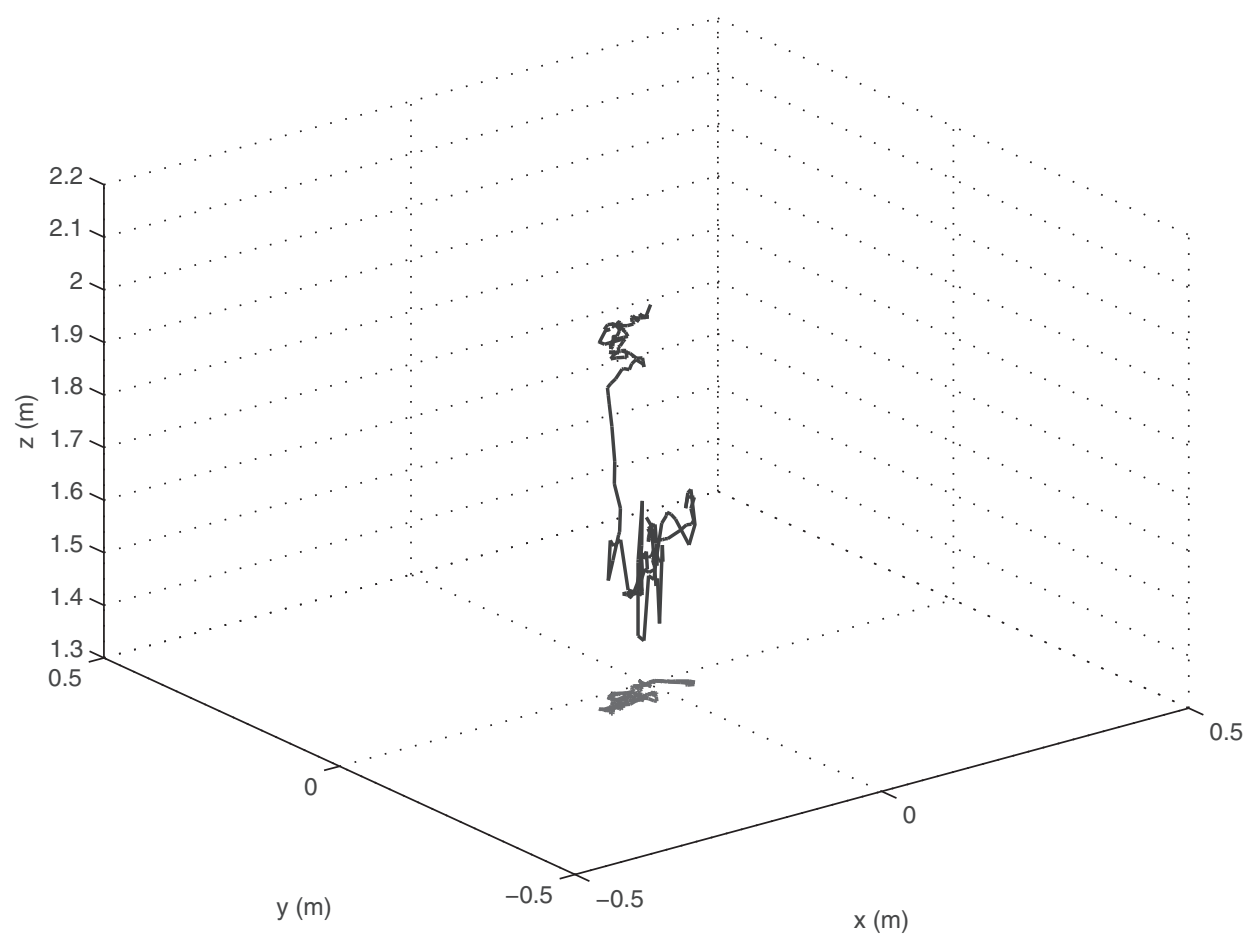

Fig. 8 Measured flight path of the quadrotor's because of a position hold and an altitude set-point command 

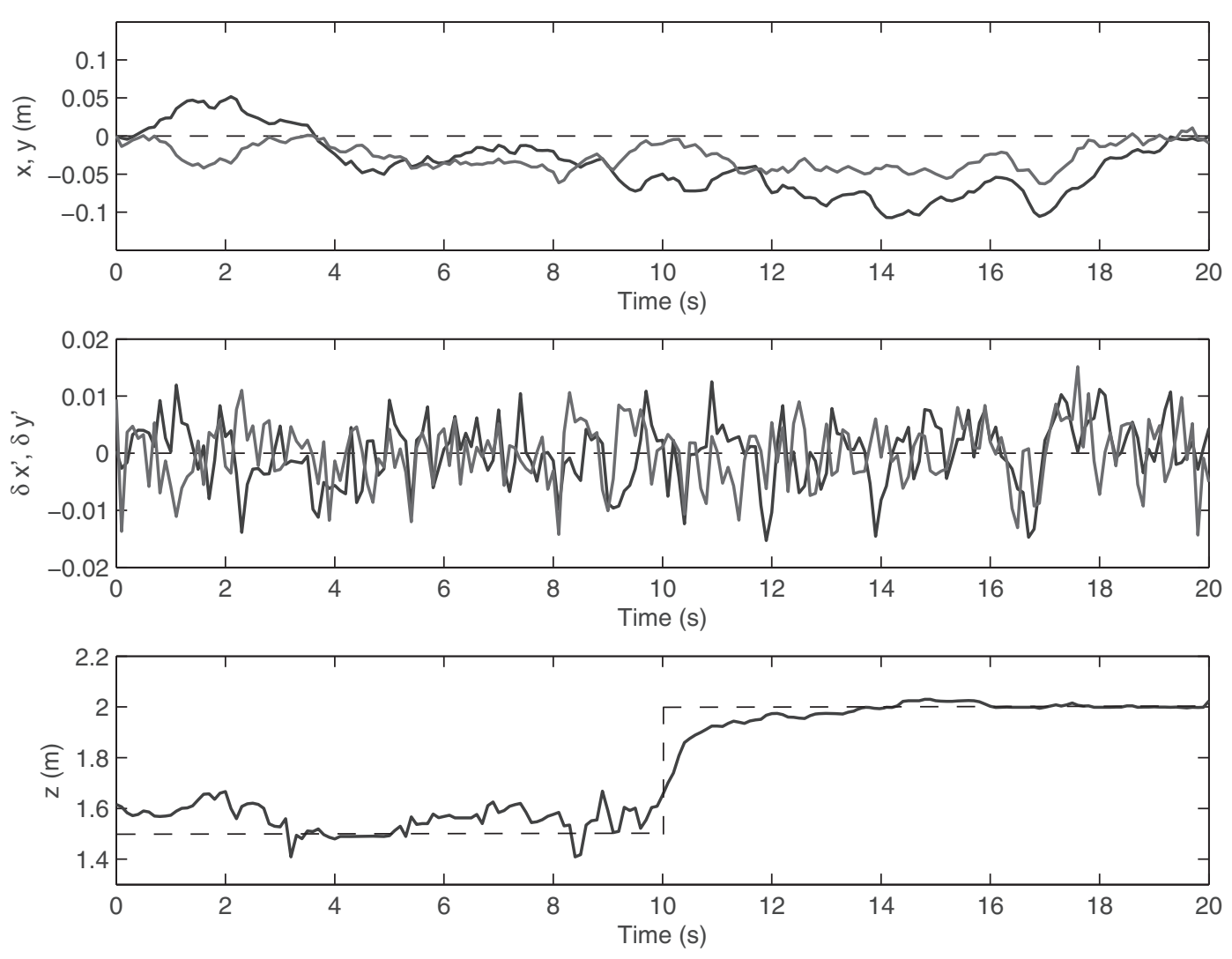

Fig.9 Estimated $x, y$ horizontal position, optic-flow measurements and altitude data of the quadrotor's flight

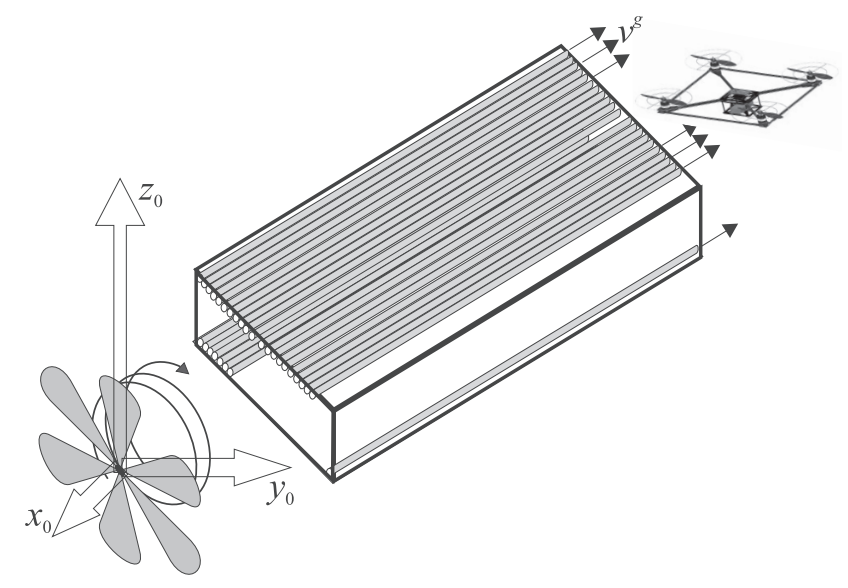

Fig. 10 Wind-gust generating set-up

both to the fact that there are existing measurement errors and the fact that the performance decreases when velocity set-point varies.

The next test-case is related to hovering response in order to examine the attitude regulation and altitude control performance of the proposed controller. The corresponding results of the hovering response are illustrated in Figs. 16 and 17.

The MSEs for the roll, pitch and yaw angles were $E_{\phi}^{\mathrm{mse}}=1.5924 \times 10^{-4}, E_{\theta}^{\mathrm{mse}}=2.2372 \times 10^{-4}, E_{\psi}^{\mathrm{mse}}=$ $4.3998 \times 10^{-4}$ and $E_{\phi}^{\mathrm{mse}}=0.0013, E_{\theta}^{\mathrm{mse}}=5.0558 \times 10^{-4}$, $E_{\psi}^{\mathrm{mse}}=0.0011$ in the absence and under the presence of wind gusts, respectively.

The performance of the attitude controller was further examined commanding the system to perform attitude

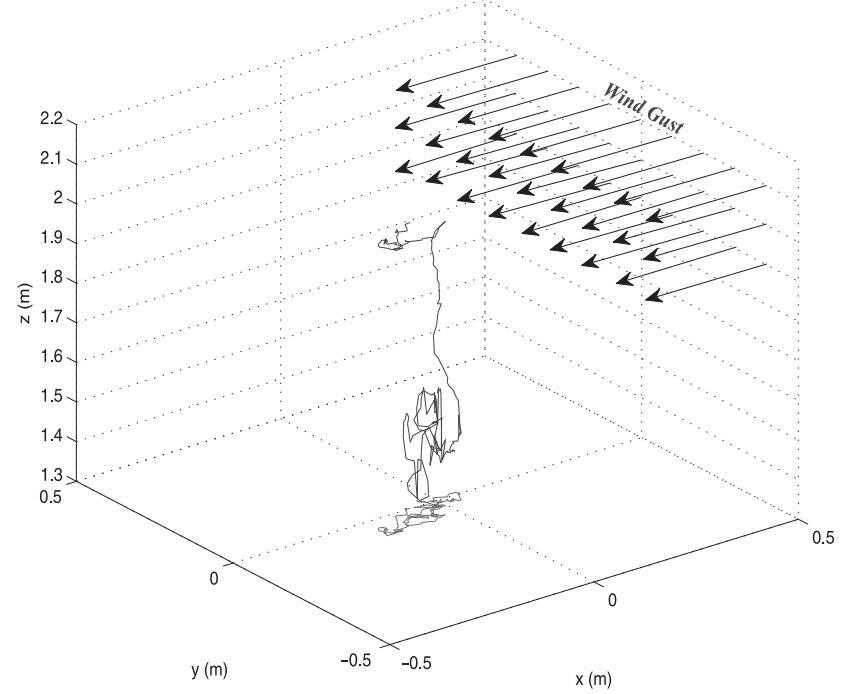

Fig. 11 Quadrotor's path in set-point altitude/hold-position control under the influence of wind gust

regulation starting from extreme initial conditions. Specifically, the initial conditions were $\left[\phi_{0^{+}}=-19.25^{\circ}, \theta_{0^{+}}=\right.$ $0.86^{\circ}, \psi_{0^{+}}=-13.24^{\circ}$ ]. The recorded results, shown in Fig. 18, show the high performance of the attitude controller both in the sense of accuracy and speed of response. Additionally, the switching among the different attitude PWA representations for the specific maneuver combined with the attitude rates are illustrated in Fig. 19.

As a final test-case, the tracking response of the attitude controller for rapidly varying reference signal at roll and pitch is presented in Fig. 20. Note that the reference signal 

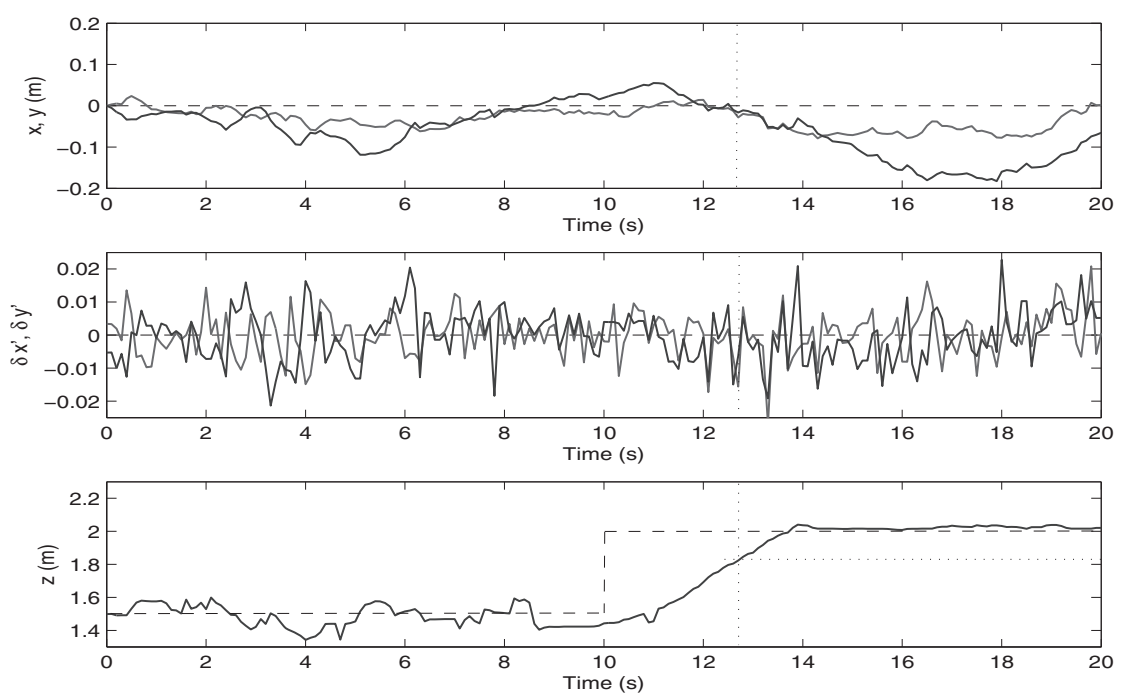

Fig. 12 Estimated $x, y$ horizontal position, optic-flow measurements and altitude data of the quadrotor's flight under the presence of a directional wind gust

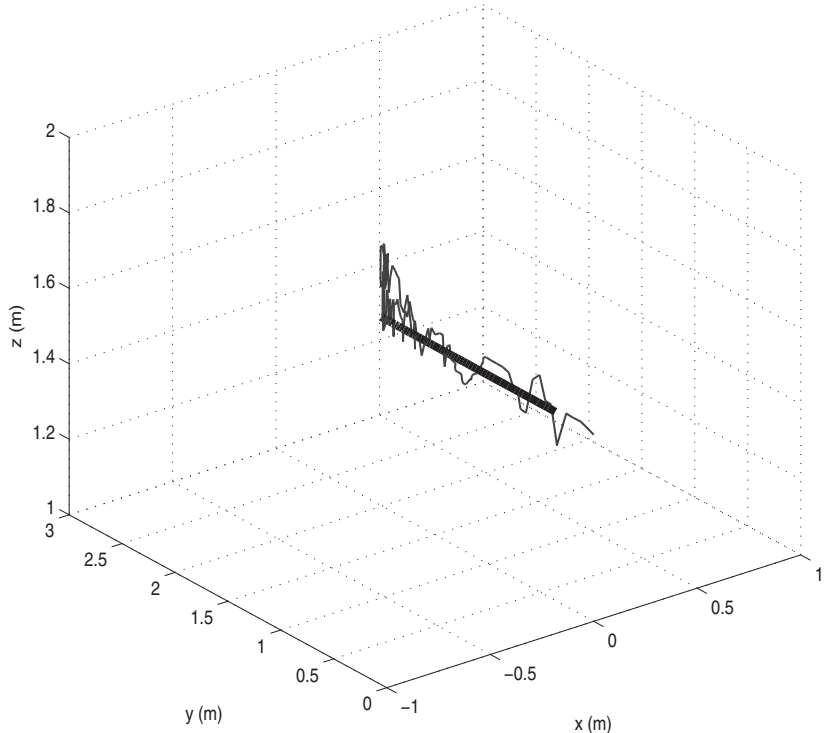

Fig. 13 Experimental flight path for translational set-point trajectory
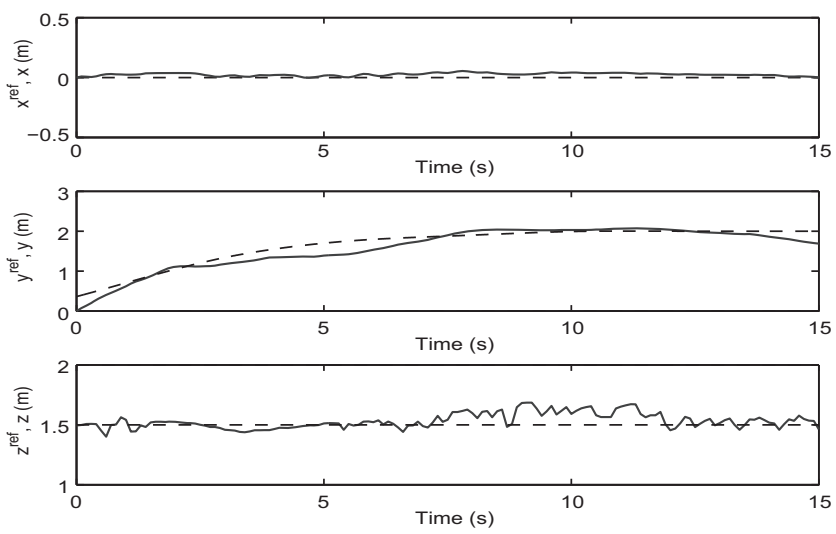

Fig. 14 Estimated $x, y, z$ position flight path of the quadrotor's flight for translational set-point trajectory

contains regions where the reference instantaneously goes to zero so discontinuities are also present. From the figure and the noted MSEs $\left(\mathrm{MSE}_{\phi}=6.3 \times 10^{-4}\right.$ and $\mathrm{MSE}_{\theta}=5.6 \times$

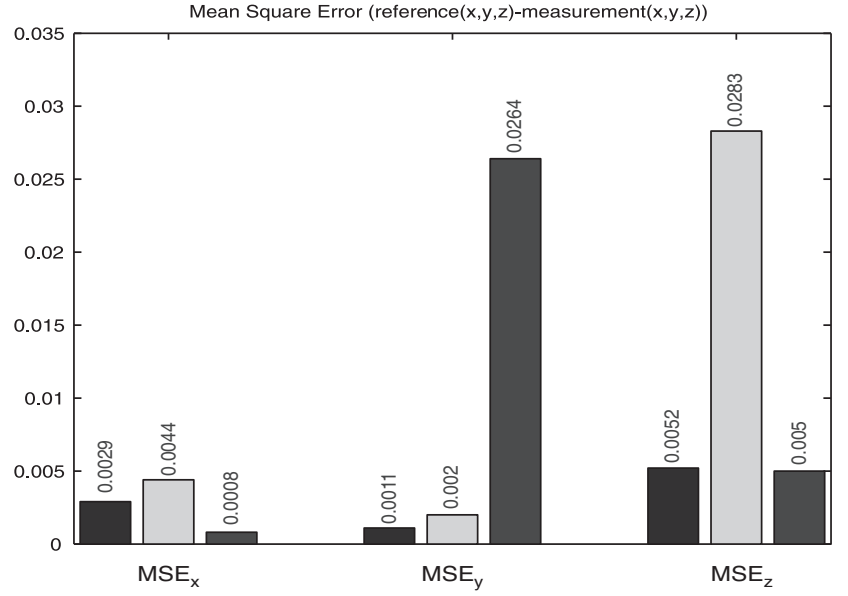

Fig. 15 MSE for x,y,z measurements for the aforementioned three experimental test-cases: at each set of three bar plots, the left bar refers to position hold in the absence of wind gusts, the middle bar refers to position hold under the presence of wind gusts and the right bar refers to trajectory following

$10^{-4}$ ) it is clearly shown that the proposed attitude control achieves accurate reference tracking, which is critical both for precise position control and disturbance attenuation.

In order to further justify the performance capabilities of the attitude controller in tracking rapid changes, provided by the position controller, the comparison of the fast Fourier transforms (FFT) of the reference and output signals, as well as, the coherence of these two signals for both roll and pitch motions are being provided in Fig. 21. The coherence of the signals is formulated as

$$
C_{r_{\mathrm{s}}, o_{\mathrm{s}}}(f)=\frac{\left|P_{r_{\mathrm{s}}, o_{\mathrm{s}}}(f)\right|^{2}}{\left|P_{r_{\mathrm{s}} r_{\mathrm{s}}}(f)\right|\left|P_{o_{\mathrm{s}} o_{\mathrm{s}}}(f)\right|}
$$

where $r_{\mathrm{s}}$ represents the reference signal, $o_{\mathrm{s}}$ the output signal, $P_{r_{\mathrm{s}} r_{\mathrm{s}}}$ and $P_{o_{\mathrm{s}} o_{\mathrm{s}}}$ is the power spectral density of the reference and output signals and $P_{r_{\mathrm{s}}, O_{\mathrm{s}}}$ is the cross power spectral density. The coherence is computed over the frequencies of the hanning-windowed signals provided by the FFT length and has been used as an additional intuitive metric [38]. 

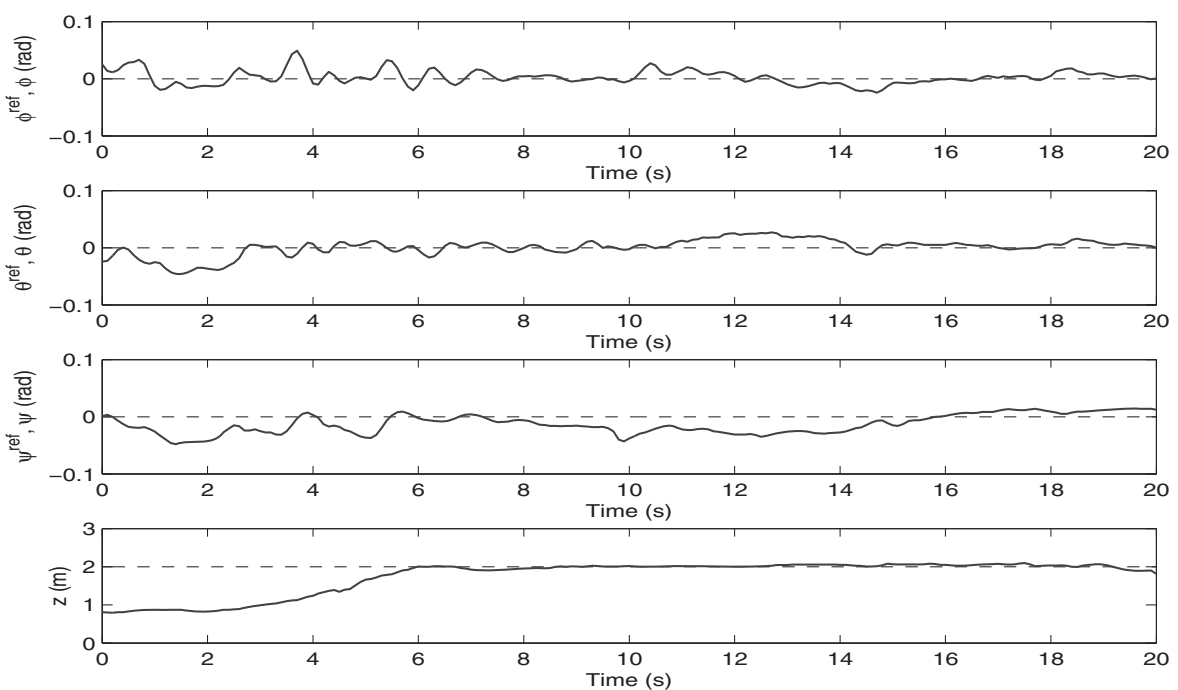

Fig. 16 Quadrotor altitude and attitude response for hovering trajectory in the absence of wind disturbances
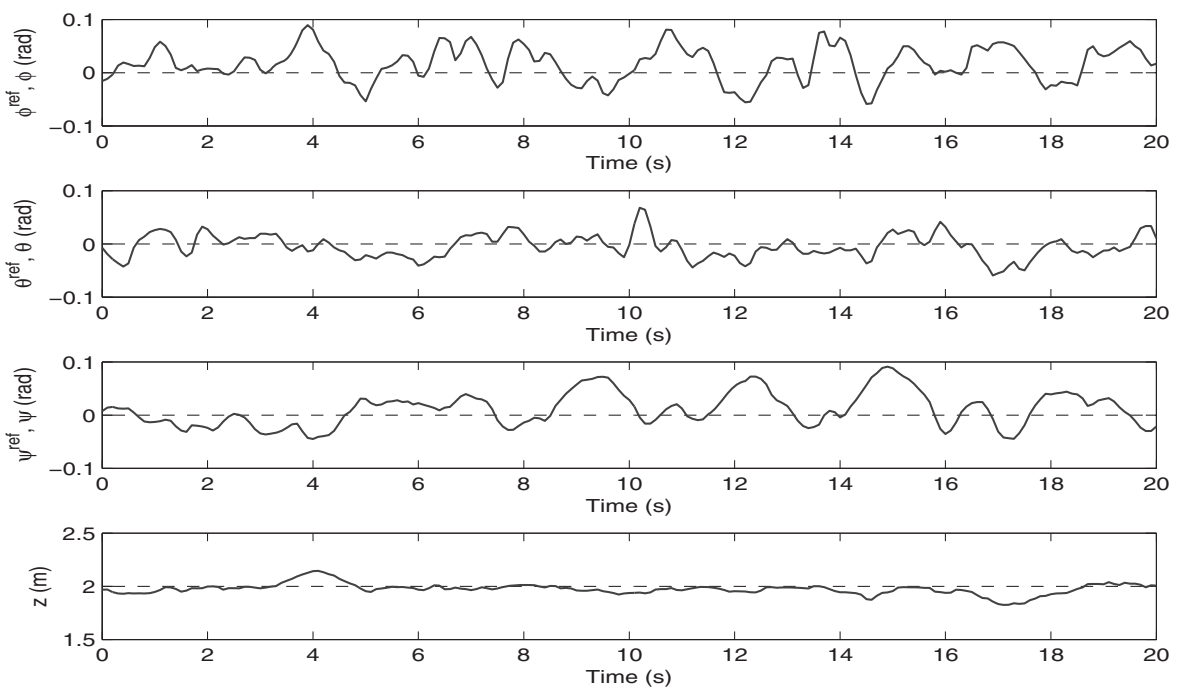

Fig. 17 Quadrotor altitude and attitude response for hovering trajectory under the presence of forcible wind gusts
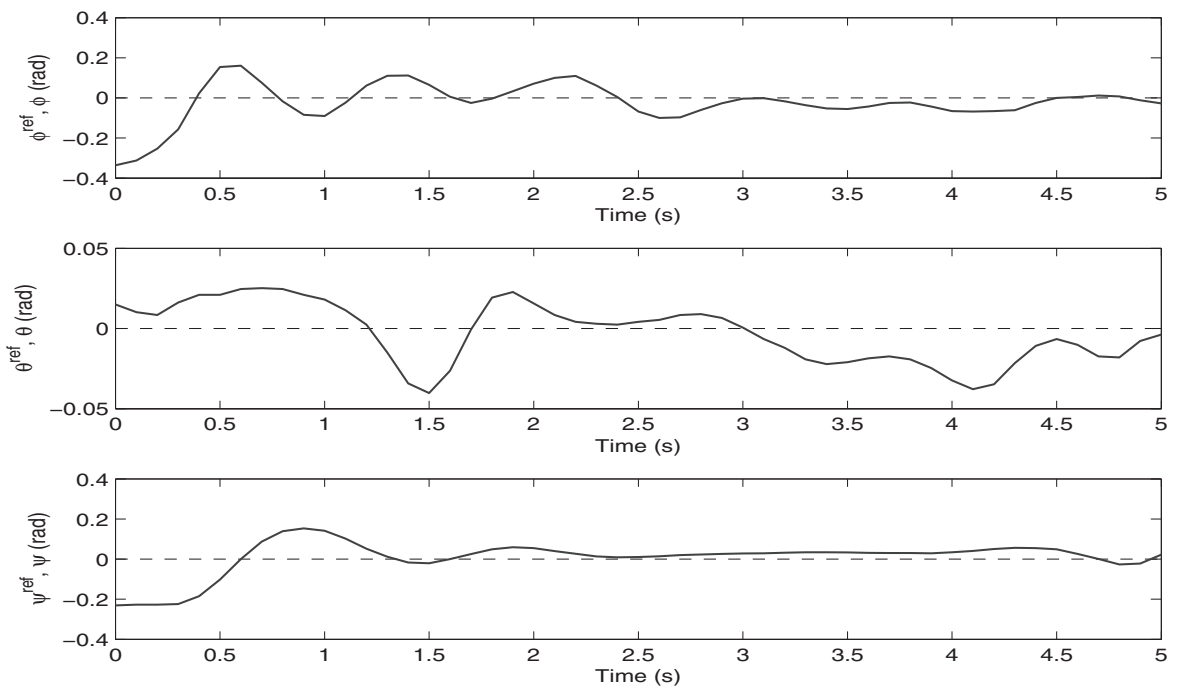

Fig. 18 Quadrotor attitude regulation response 

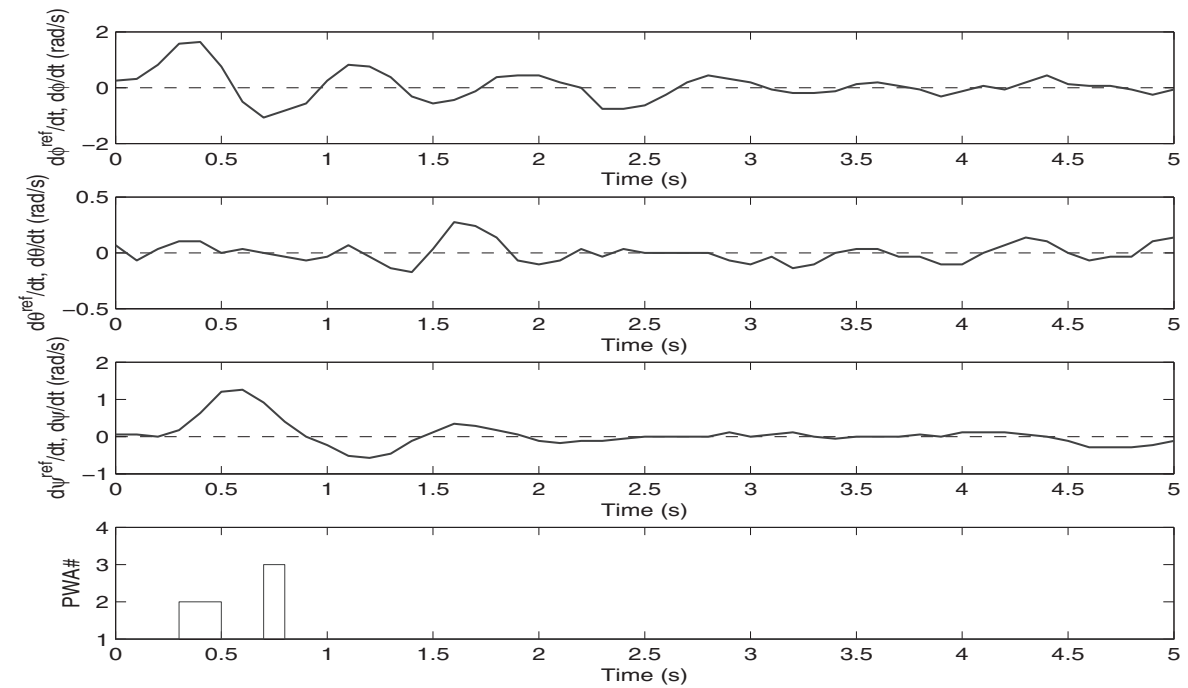

Fig. 19 Roll, pitch and yaw rates and PWA switching during attitude regulation response
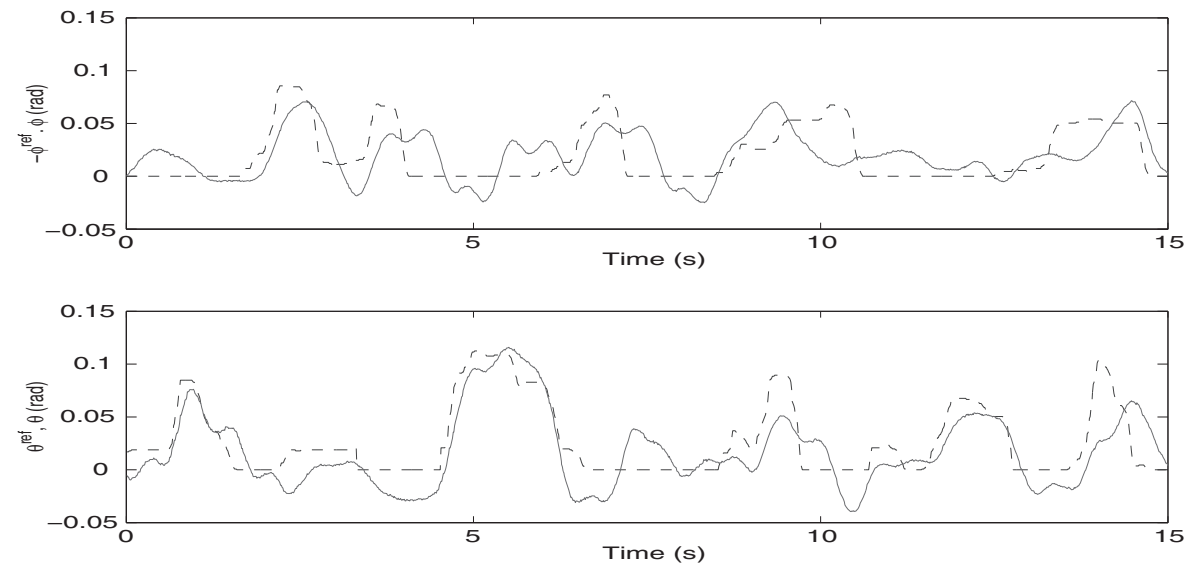

Fig. 20 Roll, pitch reference tracking response

Mean square errors are, $\mathrm{MSE}_{\phi}=6.3 \times 10^{-4}$ and $\mathrm{MSE}_{\theta}=5.6 \times 10^{-4}$
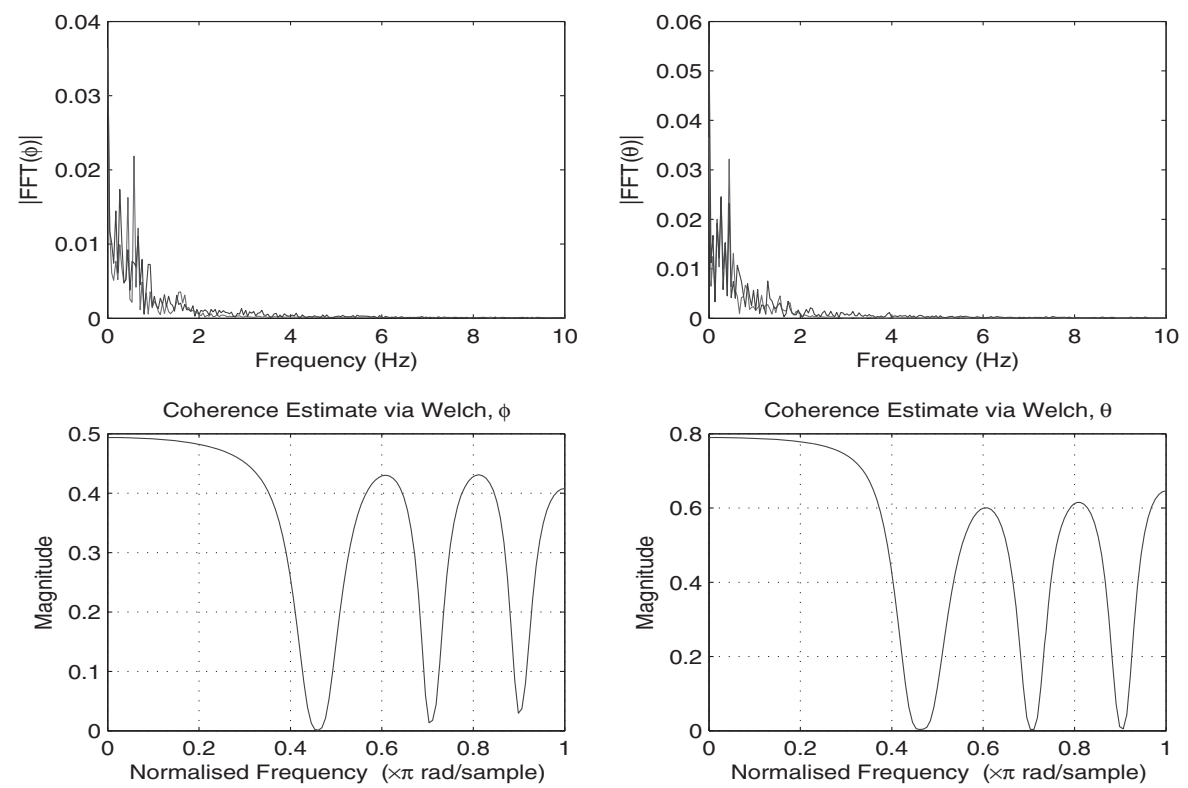

Fig. 21 FFTs of the roll-pitch reference signals and output responses and coherence metric for these two motions 
As it has been presented in the results obtained from both the MSEs and the FFT analysis, the tracking performance is accurate despite the rapid changes of the input.

From the presented experimental results it shown that the switching model predictive controller manages to achieve precise position control and attitude tracking even for aggressive attitude manoeuvres and provides effective attenuation of the effects induced by atmospheric perturbations. Overall, the SMPC-structure presented in this paper is promising in the quadrotor control problem, since in comparison with existing techniques it takes into account: (a) the actuator saturation and state constraints, (b) the effect of disturbances in the controller design phase, and (c) the larger flight envelope as a result of the multiple linearisation points.

\section{Conclusions}

In this paper, a switching model predictive position and attitude controller for a prototype unmanned quadrotor subject to wind gusts was presented. The main contributions of the suggested control approach include: (a) the development of a model predictive controller, computed over a set of PWA models of the attitude, altitude and horizontal dynamics, (b) the consideration of the effects of the applied atmospheric disturbances in the control computation, (c) the integration of the electro-mechanical and flight constraints of the system in order to produce efficient control actions, and (d) the switching among the several PWA models in order to cover a larger part of the system's flight envelope. In the developed quadrotor prototype attention was paid to the development of complete autonomous indoor state estimation based on sensor fusion strategies from data obtained from an IMU, a sonar and a vision system implementing an optic-flow algorithm. Finally, the high overall efficiency of the proposed control strategy was verified in extended experimental studies including position tracking, hovering, aggressive attitude regulation manoeuvres and forcible wind-gust attenuation.

\section{Acknowledgments}

This research has been co-financed by the European Union (European Social Fund - ESF) and Greek national funds through the Operational Program 'Education and Lifelong Learning' of the National Strategic Reference Framework (NSRF) - Research Funding Program: Heracleitus II. Investing in knowledge society through the European Social Fund. Project number: 12-260-6.

\section{References}

1 Ryan, A., Hedrick, J.: 'A mode-switching path planner for UAVassisted search and rescue'. 44th IEEE Conf. Decision and Control, 2005 European Control Conf., CDC-ECC '05, Seville, Spain, 2005, pp. 1471-1476

2 Alexis, K., Nikolakopoulos, G., Tzes, A., Dritsas, L.: 'Coordination of helicopter UAVs for aerial forest-fire surveillance', in 'Applications of intelligent control to engineering systems' (Springer, The Netherlands, 2009), pp. 169-193

3 Sarris, Z.: 'Survey of UAV applications in civil markets'. Mediterranean Conf. on Control and Automation, Ancona, Italy, 2001

4 Caprari, G., Breitenmoser, A., Fischer, W. et al.: 'Highly compact robots for inspection of power plants', J. Field Robot., 2012, 29, (1), pp. $47-68$
5 Herwitz, S.R., Johnson, L.F., Dunagan, S.E. et al.: 'Imaging from an unmanned aerial vehicle: agricultural surveillance and decision support', Comput. Electron. Agric., 2004, 44, (1), pp. 49-61

6 Committee on Autonomous Vehicles in Support of Naval Operations, National Research Council: 'Autonomous vehicles in support of naval operations' (Naval Studies Board, Washington DC, USA, 2005)

7 Gray, S.: 'Cooperation between UAVs in search and destroy mission'. American Institute of Aeronautics and Astronautics (AIAA) Guidance, Navigation, and Control Conf. and Exhibit, Austin, USA, 2003

8 Girard, A., Howell, A., Hedrick, J.: 'Border patrol and surveillance missions using multiple unmanned air vehicles'. 43rd IEEE Conf. on Decision and Control, Atlantis, Paradise Island, Bahamas, December 2004, vol. 1, pp. 620-625

9 Murphy, D., Cycon, J.: 'Applications for mini VTOL UAV for law enforcement'. Information and Training Technologies for Law Enforcement, Boston, MA, USA, November 1998

10 Bouabdallah, S., Noth, A., Siegwart, R.: 'PID vs LQ control techniques applied to an indoor micro quadrotor'. Proc. 2004 IEEE/RSJ Int. Conf. on Intelligent Robots and Systems, 2004, (IROS 2004), 2004, vol. 3, pp. 2451-2456

11 Alexis, K., Nikolakopoulos, G., Tzes, A.: 'Autonomous quadrotor position and attitude PID/PIDD control in GPS-denied environments', Int. Rev. Autom. Control, 2011, 3, pp. 421-430.

12 Hoffmann, G.M., Huang, H., Waslander, S.L., Tomlin, C.J.: 'Quadrotor helicopter flight dynamics and control: theory and experiment'. Proc. Guidance, Navigation, and Control Conf., 2007

13 Benallegue, A., Mokhtari, A., Fridman, L.: 'Feedback linearization and high order sliding mode observer for a quadrotor UAV'. Int. Workshop Variable Structure Systems (VSS'06), Alghero, Sardinia, 2006, pp. $365-372$

14 Bouabdallah, S., Siegwart, R.: 'Full control of a quadrotor'. IEEE/RSJ Int. Conf. on Intelligent Robots and Systems, 2007, IROS 2007, San Diego, CA, USA, 2007, pp. 153-158

15 Zuo, Z.: 'Trajectory tracking control design with command-filtered compensation for a quadrotor', IET Control Theory Appl., 2010, 4, (11), pp. 2343-2355

16 Das, A., Lewis, F., Subbarao, K.: 'Backstepping approach for controlling a quadrotor using lagrange form dynamics', J. Intell. Robot. Syst., 2009, 56, pp. 127-151

17 Raffo, G., Ortega, M., Rubio, F.: 'An integral predictive/nonlinear control structure for a quadrotor helicopter', Automatica, 2010, 46, 1, pp. 29-39

18 Das, A., Subbarao, K., Lewis, F.: 'Dynamic inversion with zerodynamics stabilisation for quadrotor control', IET Control Theory Appl., 2009, 3, pp. 303-314

19 Alexis, K., Nikolakopoulos, G., Tzes, A.: 'Design and experimental verification of a constrained finite time optimal control scheme for the attitude control of a quadrotor helicopter subject to wind gusts'. 2010 Int. Conf. on Robotics and Automation, Anchorage, AK, USA, 2010, pp. $1636-1641$

20 Alexis, K., Nikolakopoulos, G., Tzes, A.: 'Constrained optimal attitude control of a quadrotor helicopter subject to wind-gusts: experimental studies'. American Control Conf. '10, Baltimore, USA, 2010, pp. 4451-4455

21 Alexis, K., Nikolakopoulos, G., Tzes, A.: 'Switching model predictive attitude control for a quadrotor helicopter subject to atmospheric disturbances', Control Eng. Pract., 2011, 19, (10), pp. 1195-1207

22 Costelo, M.F., 'A theory of the analysis of rotorcraft operation in atmospheric turbulence'. $\mathrm{PhD}$ thesis, School of Aerospace Engineering, Georgia Institute of Technology, 1992

23 Yang, X., Pota, H., Garrat, M.: 'Design of a gust-attenuation controller for landing operations of unmanned autonomous helicopters'. 18th IEEE Int. Conf. on Control Applications, Saint Petersburg, Russia, July 2009, pp. 1300-1305

24 Gonzalez, A., Marchetti, J., Odloak, D.: 'Robust model predictive control with zone control', IET Control Theory Appl., 2009, 3, (1), pp. 121-135

25 Poursafar, N., Taghirad, H., Haeri, M.: 'Model predictive control of non-linear discrete time systems: a linear matrix inequality approach', IET Control Theory Appl., 2010, 4, (10), pp. 1922-1932

26 Camacho, E., Bordons, C.: 'Model predictive control' (Springer, 2003)

27 Bouabdallah, S.: 'Design and control of quadrotors with application to autonomous flying'. PhD thesis, STI School of Engineering, EPFL, Lausanee, 2007

28 Xsens: 'Xsens MTi-G', http://www.xsens.com/en/general/mti-g, 2008

29 Centeye: 'Tam2 and Tam4 vision chips', http://centeye.com/ technology/vision-chips, WA, USA, February 2011

30 Srinivasan, M.V.: 'An image-interpolation technique for the computation of optic flow and egomotion', Biol. Cybern., 1994, 71, (5), pp. 401-415 


\section{www.ietdl.org}

31 Zufferey, J.-C.: 'Bio-inspired flying robots - experimental synthesis of autonomous indoor flyers' (EPFL Press, 2008)

32 Simon, D.: 'Optimal state estimation: Kalman, $\mathrm{H}$ infinity and nonlinear approaches' (John Wiley and Sons, Hoboken, NJ, USA, 2006)

33 Lange, S., Sunderhauf, N., Protzel, P.: 'A vision based onboard approach for landing and position control of an autonomous multirotor UAV in GPS-denied environments'. International Conference on Advanced Robotics (ICAR), Munich Marriot Hotel, Germany, June 22-24, 2009

34 Moore, R.: 'Methods and applications of interval analysis' (SIAM Bookmart, Philadelphia, 1979)
35 Zhang, R., Quan, Q., Cai, K.-Y.: 'Attitude control of a quadrotor aircraft subject to a class of time-varying disturbances', IET Control Theory Appl., 2011, 5, (9), pp. 1140-1146

36 Abraham, R.H., Bachrach Roy, N.: 'Autonomous flight in unstructured and unkown indoor environments'. European Micro Aerial Vehicle Conf. and Flight Competition (EMAV), ASTI, D-CIS LAB, TUDelft, THALES, The Netherlands, 14-17 September 2009

37 Huang, H.: 'Aerodynamics and control of autonomous quadrotor helicopters in aggressive maneuvering'. Int. Conf. on Robotics and Automation, 2009, Kobe, Japan

38 Mettler, B.: 'Identification modeling and characteristics of miniature rotorcraft' (Kluwer Academic Press, 2003) 